\title{
Search for a charged Higgs boson decaying into top and bottom quarks in events with electrons or muons in proton-proton collisions at $\sqrt{\mathrm{s}}=13 \mathrm{TeV}$
}

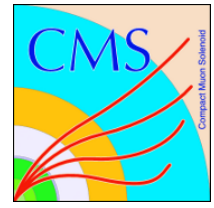

\section{The CMS collaboration}

E-mail: cms-publication-committee-chair@cern.ch

ABSTRACT: A search is presented for a charged Higgs boson heavier than the top quark, produced in association with a top quark, or with a top and a bottom quark, and decaying into a top-bottom quark-antiquark pair. The search is performed using proton-proton collision data collected by the CMS experiment at the LHC at a center-of-mass energy of $13 \mathrm{TeV}$, corresponding to an integrated luminosity of $35.9 \mathrm{fb}^{-1}$. Events are selected by the presence of a single isolated charged lepton (electron or muon) or an opposite-sign dilepton (electron or muon) pair, categorized according to the jet multiplicity and the number of jets identified as originating from $b$ quarks. Multivariate analysis techniques are used to enhance the discrimination between signal and background in each category. The data are compatible with the standard model, and 95\% confidence level upper limits of 9.6-0.01 pb are set on the charged Higgs boson production cross section times branching fraction to a top-bottom quark-antiquark pair, for charged Higgs boson mass hypotheses ranging from $200 \mathrm{GeV}$ to $3 \mathrm{TeV}$. The upper limits are interpreted in different minimal supersymmetric extensions of the standard model.

KEYwords: Hadron-Hadron scattering (experiments), Higgs physics, Supersymmetry

ArXIV EPRINT: 1908.09206 


\section{Contents}

1 Introduction 1

2 The CMS detector 3

3 Event simulation $\quad 4$

4 Event reconstruction $\quad 5$

$\begin{array}{lll}5 & \text { Event selection and classification } & 7\end{array}$

6 Background estimation and systematic uncertainties 11

$\begin{array}{llr}7 & \text { Results } & 14\end{array}$

$\begin{array}{lll}8 & \text { Summary } & 17\end{array}$

$\begin{array}{lr}\text { The CMS collaboration } & 28\end{array}$

\section{Introduction}

Since the discovery of a Higgs boson [1-3] with a mass of $125 \mathrm{GeV}[4,5]$, the ATLAS and CMS collaborations have actively searched for additional neutral and charged Higgs bosons. Most theories beyond the standard model (SM) of particle physics enrich the SM Higgs sector; a simple extension is the assumption of the existence of two Higgs doublets [6-9]. Such models are collectively labeled as two-Higgs-doublet models (2HDM), and are further classified into four categories according to the couplings of the doublets to fermions. In Type-I models, only one doublet couples to fermions, while in Type-II models one doublet couples to the up-type quarks and the other to the down-type quarks and the charged leptons. In lepton-specific models one doublet couples only to the leptonic sector and the other couples to quarks, while in flipped models the first doublet couples specifically to the down-type quarks and the second one to the up-type quarks and charged leptons.

The two-doublet structure of the 2HDM Higgs sector gives rise to five physical Higgs bosons through spontaneous symmetry breaking: a charged pair $\left(\mathrm{H}^{ \pm}\right)$and three neutral bosons, namely the light $(\mathrm{h})$ and heavy $(\mathrm{H})$ scalar Higgs bosons, and one pseudoscalar boson (A). Supersymmetric (SUSY) models have a Higgs sector based on 2HDMs [1015]. Among the SUSY models, a popular one is the minimal supersymmetric extension to the SM (MSSM) [16, 17], whose Higgs sector is described by a Type-II 2HDM. In the MSSM, the production and decay of these particles are described at tree level by two free parameters, which can be chosen as the mass of the charged Higgs boson $\left(m_{\mathrm{H}^{ \pm}}\right)$and 

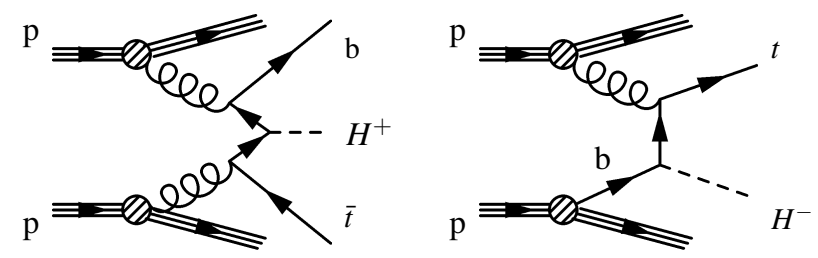

Figure 1. Feynman diagrams for the production of a heavy charged Higgs boson in the four-flavor scheme (4FS, left) and in the five-flavor scheme (5FS, right).

the ratio of the vacuum expectation values of the neutral components of the two Higgs doublets $(\tan \beta)$.

Some variants of the $2 \mathrm{HDM}$ achieve consistency with the $125 \mathrm{GeV}$ Higgs boson via a Gildener-Weinberg scalon scenario which stabilizes the Higgs boson mass and alignment [18].

Charged Higgs bosons with a mass below the top quark mass are dominantly produced in top quark decays, whereas charged Higgs bosons with a mass larger than the top quark mass are produced in association with a top quark. Charged Higgs boson production at finite order in perturbation theory is accomplished in association with a top and a bottom quark in the so-called four-flavor scheme (4FS) and in association with a top quark in the five-flavor scheme (5FS) [19], as illustrated in figure 1.

In this paper, only charged Higgs bosons with a mass larger than the mass of the top quark (heavy charged Higgs bosons) are considered, and charge-conjugate processes are implied. The signal is produced in the $4 \mathrm{FS}$, and the eventual presence of a $5 \mathrm{FS}$ production is accounted for in the search region definition. The normalization of the signal processes accounts for both the 4FS and the 5FS.

The decay of a heavy charged Higgs boson can occur through several channels, among them $\mathrm{H}^{+} \rightarrow \tau^{+} v_{\tau}$ and $\mathrm{H}^{+} \rightarrow \mathrm{t} \overline{\mathrm{b}}$ have the highest branching fractions, respectively at low (about $200 \mathrm{GeV}$ ) and high (about $1 \mathrm{TeV}) m_{\mathrm{H}^{ \pm}}$for a large range of $\tan \beta$ values and a large variety of theoretical models [20].

The detection of a charged Higgs boson would unequivocally point to physics beyond the SM. Model-independent searches for charged Higgs bosons are of utmost interest for the CERN LHC program because they allow one to disentangle the Higgs sector physics from the specificity and complexity of the theoretical model by assuming unity branching fraction in each mode.

Direct searches for charged Higgs bosons have been performed by the CERN LEP and the Fermilab Tevatron experiments, and indirect constraints on $\mathrm{H}^{ \pm}$production have been set from flavor physics measurements [21-30]. Searches for a charged Higgs boson decaying into a top and a bottom quark have been performed by the D0, ATLAS, and CMS collaborations in proton-antiproton collisions at a center-of-mass energy of $\sqrt{s}=1.96 \mathrm{TeV}$ [31] and in proton-proton (pp) collisions at $\sqrt{s}=8 \mathrm{TeV}[32,33]$ and $\sqrt{s}=13 \mathrm{TeV}$ [34]. In this paper we improve the sensitivity to model-independent production of a charged Higgs boson, as well as the sensitivity to relevant MSSM scenarios. The ATLAS and CMS collaborations have also conducted searches for the production of a charged Higgs boson in

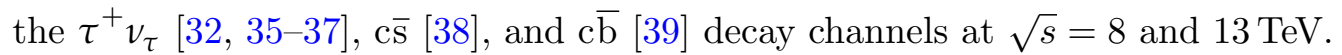


Searches for charged Higgs bosons produced via vector boson fusion and decaying into $\mathrm{W}$ and $\mathrm{Z}$ bosons, as predicted by models containing Higgs triplets [40-42], and searches for additional neutral heavy Higgs bosons decaying to a pair of third-generation fermions $\mathrm{t} \overline{\mathrm{t}}, \mathrm{b} \overline{\mathrm{b}}$, and $\tau^{+} \tau^{-}[41,43-46]$ extend the program of the ATLAS and CMS collaborations to elucidate the extended Higgs sector beyond the SM.

This paper describes a search for a heavy charged Higgs boson produced in association with a top quark or with a top and a bottom quark and decaying into a top and a bottom quark performed using pp collision data collected at $\sqrt{s}=13 \mathrm{TeV}$ in 2016 . The data correspond to an integrated luminosity of $35.9 \mathrm{fb}^{-1}$. The final state contains two $\mathrm{W}$ bosons, one from the decay chain of the heavy charged Higgs boson and the other from the decay of the associated top quark. One or both of the $\mathrm{W}$ bosons can decay into leptons, producing single-lepton and dilepton final states, respectively. The leptonic decays of tau leptons from the $\mathrm{W}$ boson decay are considered as well. The single-lepton final state is characterized by the presence of one isolated lepton $(\mathrm{e}, \mu)$ that is used to trigger the event, while the dilepton final state contains events with two isolated opposite-sign leptons $\left(\mathrm{e}^{+} \mathrm{e}^{-}, \mathrm{e}^{ \pm} \mu^{\mp}, \mu^{+} \mu^{-}\right)$. This leads to the suppression of several backgrounds. The signal process $\left(\overline{\mathrm{t}} \mathrm{bH}^{+}+\overline{\mathrm{t}} \mathrm{H}^{+}\right)$has furthermore a large b jet multiplicity; an additional classification of the events is therefore achieved based on the number of jets identified as originating from $\mathrm{b}$ quarks.

Multivariate analysis (MVA) techniques are used to enhance the discrimination between signal and background. Signal-rich regions are analyzed together with signal-depleted regions in a maximum likelihood fit to the MVA classifier outputs, which simultaneously determines the contributions from the $\overline{\mathrm{t}} \mathrm{bH} \mathrm{H}^{+}+\overline{\mathrm{t}} \mathrm{H}^{+}$signal and the backgrounds.

Model-independent upper limits on the product of the charged Higgs boson production cross section and the branching fraction into a top-bottom quark-antiquark pair, $\sigma_{\mathrm{H}^{ \pm}} \mathcal{B}\left(\mathrm{H}^{ \pm} \rightarrow \mathrm{tb}\right)=\sigma\left(\mathrm{pp} \rightarrow \mathrm{H}^{+} \overline{\mathrm{t}} \mathrm{b}+\mathrm{pp} \rightarrow \mathrm{H}^{+} \overline{\mathrm{t}}\right) \mathcal{B}\left(\mathrm{H}^{+} \rightarrow \mathrm{t} \overline{\mathrm{b}}\right)+\sigma\left(\mathrm{pp} \rightarrow \mathrm{H}^{-} \mathrm{t} \overline{\mathrm{b}}+\mathrm{pp} \rightarrow\right.$ $\left.\mathrm{H}^{-} \mathrm{t}\right) \mathcal{B}\left(\mathrm{H}^{-} \rightarrow \overline{\mathrm{t}} \mathrm{b}\right)$, as a function of $m_{\mathrm{H}^{ \pm}}$, are presented in this paper. Results are also interpreted in specific MSSM benchmark scenarios, where many free parameters of the model are fixed to values corresponding to interesting phenomenological assumptions.

\section{The CMS detector}

The central feature of the CMS apparatus is a superconducting solenoid of $6 \mathrm{~m}$ internal diameter, providing a magnetic field of $3.8 \mathrm{~T}$. Within the solenoid volume are a silicon pixel and strip tracker, a lead tungstate crystal electromagnetic calorimeter (ECAL), and a brass and scintillator hadron calorimeter (HCAL), each composed by a barrel and two endcap sections. Forward calorimeters extend the pseudorapidity $(\eta)$ coverage provided by the barrel and endcap detectors. Muons are detected in gas-ionization chambers embedded in the steel flux-return yoke outside the solenoid. Events of interest are selected using a two-tiered trigger system [47]. The first level, composed by specialized hardware processors, uses information from the calorimeters and muon detectors, while the second level consists of a farm of processors running a version of the full event reconstruction software optimized for fast processing. A more detailed description of the CMS detector, together with a 
definition of the coordinate system used and the relevant kinematic variables, can be found in ref. [48].

\section{Event simulation}

Signal events are simulated using the MADGRAPH5_aMC@NLO 2.3.3 [49] generator at nextto-leading order (NLO) precision in perturbative quantum chromodynamics (QCD) using the $4 \mathrm{FS}$ for a range of $m_{\mathrm{H}^{ \pm}}$hypotheses between 200 and $3000 \mathrm{GeV}$; the complete list of masses is $[200,220,250,300,350,400,500,650,800,1000,1500,2000,2500,3000] \mathrm{GeV}$. The $4 \mathrm{FS}$ is expected to provide a better description of the observables, while shape effects from 5FS production are expected to be negligible, because eventual additional b quarks would be radiated with low transverse momentum by the beam remnants [20].

Normalization effects induced by the presence of 5FS are accounted for by computing the MSSM production cross sections for the heavy charged Higgs boson signals both in the $4 \mathrm{FS}$ and 5FS; the two cross sections are then combined to obtain the total cross section using the Santander matching scheme [19] for different values of $\tan \beta$. The 4FS and 5FS cross sections differ for all mass point by about $20 \%$, and the Santander-matched cross section lies inbetween the two; typical values are of the order of $1 \mathrm{pb}$ for a mass of $200 \mathrm{GeV}$, down to about $10^{-4} \mathrm{pb}$ for a mass of $3 \mathrm{TeV}[20,50-54]$.

Branching fractions $\mathcal{B}\left(\mathrm{H}^{+} \rightarrow \mathrm{t} \overline{\mathrm{b}}\right)$ are computed in the chosen scenarios with the HDECAY 6.52 package [55]. These cross sections are used in section 7 only for the modeldependent results, and don't affect the model-independent results.

The main background to this analysis originates from SM top quark pair production. Other backgrounds are the production of $\mathrm{W}$ and $\mathrm{Z} / \gamma^{*}$ with additional jets (referred to as $\mathrm{V}+$ jets), diboson and triboson processes, single top quark production, $\mathrm{t} \overline{\mathrm{t}}$ production in association with $\mathrm{W}, \mathrm{Z}, \gamma$, or $\mathrm{H}$ bosons (collectively labeled $\mathrm{t} \overline{\mathrm{t}}+\mathrm{V}$ ), as well as four top quark production $(t \bar{t} t \bar{t})$ and $\mathrm{QCD}$ multijet events.

The $t \bar{t}, t \bar{t} H$, and single top quark events in the $t$ - and $t W$-channels are generated at NLO precision in perturbative QCD with POWHEG v2.0 [56-58].

The MadGraph5_amC@NLO 2.2.2 generator [49] is used at leading order (LO), with the MLM jet matching and merging [59], to generate vector boson events in association with jets, single top quark events in the $s$-channel, and four top quark production. The associated production of $\mathrm{t} \overline{\mathrm{t}}$ events with a vector boson and with a $\gamma$ is simulated at NLO using MADGraPh5_aMC@NLO 2.2.2 with FxFx jet matching and merging [60].

In all cases, the NNPDF3.0 [61] set of parton distribution functions (PDFs) is used, and the parton showers and hadronization processes are performed by PYTHIA 8.212 [62] with

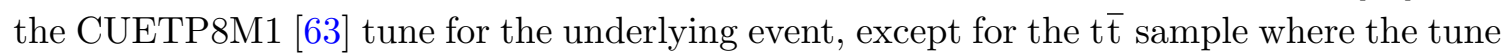
CUETP8M2T4 [64] provides a more accurate description of the kinematic distributions of the top quarks and of the jet multiplicity.

Next-to-NLO (NNLO) calculations are used to compute the cross section for the dominant $t \overline{\mathrm{t}}$ background for a top quark mass of $172.5 \mathrm{GeV}$, including resummation to nextto-next-to-leading-logarithmic accuracy [65-71]. The other backgrounds are normalized using NLO (single top quark $t$ - and $s$-channels [72, 73], t $\overline{\mathrm{t}}+\mathrm{V}$ production [74], and diboson 
production [75]), NNLO (V+jets production), and approximate NNLO (single top quark tW-channel [76]) cross sections.

The simulated $t \bar{t}$ events are further separated based on the flavor of additional jets that do not originate from the top quark decays in the event and are labeled according to their content in $\mathrm{b}$ - and c-originated hadrons. The $t \overline{\mathrm{t}}+\mathrm{b}(\mathrm{b})(\mathrm{t} \overline{\mathrm{t}}+\mathrm{c}(\mathrm{c}))$ label is attributed to the events that have at least one $b$ jet (c jet and no $b$ jet) from the event generator within the acceptance. Events that do not belong to any of the above processes are enriched in light-flavor jets and therefore denominated as $t \bar{t}+L F$. This partition of the simulated $t \bar{t}$ sample is based on matching heavy-flavor generator-level jets to the originating partons and hadrons and is introduced to account for different systematic uncertainties affecting the corresponding cross section predictions. The procedure is detailed in refs. [77, 78].

All generated events are passed through a detailed simulation of the CMS apparatus, based on GEANT4 v9.4 [79]. The effects of additional pp interactions occurring in the same or in neighboring bunch crossings (pileup) are modelled by adding simulated minimum bias events to all simulated processes. In the data collected in 2016 an average of $23 \mathrm{pp}$ interactions occurred per LHC bunch crossing. In simulation, the difference in the number of true interactions is accounted for by reweighting the simulated events to match the data in the multiplicity distribution of pileup interactions.

\section{Event reconstruction}

Events are reconstructed using the particle-flow (PF) algorithm [80], which aims to reconstruct and identify each individual particle in an event, with an optimized combination of information from the various elements of the CMS detector. The energy of photons is obtained from the ECAL measurement. The energy of electrons is determined from a combination of the electron momentum at the primary interaction vertex as determined by the tracker, the energy of the corresponding ECAL cluster, and the energy sum of all bremsstrahlung photons spatially compatible with originating from the electron track. The momentum of muons is obtained from the curvature of the corresponding track. The energy of charged hadrons is determined from a combination of their momentum measured in the tracker and the matching ECAL and HCAL energy deposits, corrected for zero-suppression effects and for the response function of the calorimeters to hadronic showers. Finally, the energy of neutral hadrons is obtained from the corresponding corrected ECAL and HCAL energy. The reconstructed vertex with the largest value of summed physics-object squared transverse momentum $\left(p_{\mathrm{T}}^{2}\right)$ is taken to be the primary pp interaction vertex [81]. The physics objects are the jets, clustered using the jet finding algorithm $[82,83]$ with the tracks assigned to the vertex as inputs, and the associated missing transverse momentum $\left(\vec{p}_{\mathrm{T}}^{\text {miss }}\right)$, taken as the negative vector sum of the $p_{\mathrm{T}}$ of those jets.

Electrons are identified using an MVA-based identification algorithm [84]. Working points are defined [85] by setting thresholds for the classifier values to mitigate efficiency losses for high- $p_{\mathrm{T}}$ electrons observed particularly in high-mass signal events; such working points are labeled Tight ( $\approx 88 \%$ efficiency for $\mathrm{t} \overline{\mathrm{t}}$ events) and Loose ( $\approx 95 \%$ efficiency for $t \bar{t}$ events). They result in an efficiency in selecting high-mass signal events of $\approx 90 \%$, ap- 
proximately flat across the electron high- $p_{\mathrm{T}}$ range. Muon identification uses the algorithm described in ref. [86] and two working points, referred to as Medium and Loose, with efficiencies of about 97 and $100 \%$, respectively. Thresholds in $p_{\mathrm{T}}$ and $\eta$ for electrons and muons depend on whether they are used for selecting or vetoing events and are detailed in section 5 .

Electrons and muons are required to be isolated from other particles. Their relative isolation is measured as the ratio between the scalar $p_{\mathrm{T}}$ sum of selected PF particles within a cone of a radius $\Delta R\left(p_{\mathrm{T}}(\ell)\right)$ and the $p_{\mathrm{T}}$ of the particle; $\Delta R$ is defined as $\sqrt{(\Delta \eta)^{2}+(\Delta \phi)^{2}}$ and $\Delta \eta$ and $\Delta \phi$ are the distances in the pseudorapidity and azimuthal angle. The $\Delta R\left(p_{\mathrm{T}}(\ell)\right)$ cone decreases with the lepton $p_{\mathrm{T}}[87,88]$ according to the formula

$$
\Delta R\left(p_{\mathrm{T}}(\ell)\right)=\frac{10 \mathrm{GeV}}{\min \left[\max \left(p_{\mathrm{T}}(\ell), 50 \mathrm{GeV}\right), 200 \mathrm{GeV}\right]} .
$$

Efficiencies in triggering, reconstruction, identification, and isolation of leptons are estimated both in data and simulation. Those efficiencies are used to determine correction factors, depending on $p_{\mathrm{T}}$ and $\eta$, and are applied to simulated events on a per-lepton basis.

Jets are reconstructed from the PF particles clustered by the anti- $k_{\mathrm{T}}$ algorithm $[82,83]$ with a clustering radius of 0.4 . To mitigate the effect of pileup interactions, charged hadrons that do not arise from the primary vertex are excluded from the clustering. Furthermore, jets originating from pileup interactions are removed by means of an MVA identification algorithm [89]. The jet momentum is then corrected in simulated events to account for multiple effects, including the extra energy clustered in jets arising from pileup. In situ measurements of the momentum balance in dijet, photon+jet, $\mathrm{Z}+$ jet, and multijet events are used to determine any residual differences between the jet energy scale in data and in simulation, and appropriate corrections are applied [90]. Jets are selected if they satisfy $p_{\mathrm{T}}>40 \mathrm{GeV}$ and $|\eta|<2.4$. Loose identification criteria are applied to the jets, in order to distinguish them from well-identified stable particles. Finally, jets are required to be separated from the selected leptons by $\Delta R>0.4$.

Jets from the hadronization of $\mathrm{b}$ quarks are identified ( $\mathrm{b}$ tagged) using the combined secondary vertex algorithm [91]. For the chosen threshold of the tagging algorithm, the mistagging probability - the fraction of jets that arise from the fragmentation of light partons $(\mathrm{u}, \mathrm{d}, \mathrm{s}$, and $\mathrm{g})$ and $\mathrm{c}$ jets misidentified by the algorithm as $\mathrm{b}$ jets - is approximately 1 and $15 \%$, respectively, while the efficiency to correctly identify a b jet is about $70 \%$. The difference in $\mathrm{b}$ tagging and mistagging efficiencies between data and simulation is corrected by applying correction factors dependent on jet $p_{\mathrm{T}}$ and $\eta$.

The missing transverse momentum vector is defined as the projection of the negative vector sum of the momenta of all reconstructed PF particles in an event onto the plane perpendicular to the beams. Its magnitude is referred to as $p_{\mathrm{T}}^{\text {miss }}$. The $\vec{p}_{\mathrm{T}}^{\text {miss }}$ reconstruction is improved by propagating the effect of the jet energy corrections to it. Further filtering algorithms are used to reject events with anomalously large $p_{\mathrm{T}}^{\text {miss }}$ resulting from instrumental effects [92].

Hadronically decaying $\tau$ leptons $\left(\tau_{\mathrm{h}}\right)$ are reconstructed using the hadron-plus-strips algorithm [93], based on the identification of the individual $\tau$ decay modes. The $\tau_{\mathrm{h}}$ candi- 
dates are required to be separated from reconstructed electrons and muons by $\Delta R>0.4$. Tau candidates are further selected by means of a multivariate discriminator combining isolation and lifetime information [93]. Jets originating from the hadronization of quarks and gluons misidentified as $\tau_{\mathrm{h}}$ are suppressed by requiring that the $\tau_{\mathrm{h}}$ candidate is isolated. The $\tau_{\mathrm{h}}$ identification efficiency depends on $p_{\mathrm{T}}^{\tau_{\mathrm{h}}}$ and $\eta^{\tau_{\mathrm{h}}}$, and is on average $50 \%$ for $p_{\mathrm{T}}^{\tau_{\mathrm{h}}}>20 \mathrm{GeV}$ with a probability of approximately $1 \%$ for hadronic jets to be misidentified as a $\tau_{\mathrm{h}}$. The isolation variable is constructed from the PF particles inside a cone of $\Delta R=0.3$. The effect of neutral $\mathrm{PF}$ candidates from pileup vertices is estimated using charged hadrons associated with those vertices and subtracted from the isolation variable.

\section{Event selection and classification}

Events are selected with single-lepton triggers characterized by transverse momentum $\left(p_{\mathrm{T}}\right)$ thresholds of 27 (24) GeV for electrons (muons). Additionally, several trigger paths with higher $p_{\mathrm{T}}$ thresholds and looser identification requirements are included to maximize efficiency for high- $p_{\mathrm{T}}$ electrons (muons), resulting in an overall efficiency in the plateau region close to 95 (100)\%. Correction factors quantifying the difference between trigger efficiencies in data and simulated events are evaluated using a tag-and-probe technique [84, 86, 94, 95].

Events are required to have at least one electron (muon) with $p_{\mathrm{T}}>35(30) \mathrm{GeV}$ satisfying tighter identification and isolation criteria than the online requirements, effectively corresponding to the saturation point of the online trigger efficiencies. As briefly discussed in section 1 , the first classification is achieved by separating the events in five single-lepton and dilepton regions $\left(\mathrm{e}^{ \pm}, \mu^{ \pm}, \mathrm{e}^{+} \mathrm{e}^{-}, \mathrm{e}^{ \pm} \mu^{\mp}, \mu^{+} \mu^{-}\right)$. In the single-lepton category, only events with exactly one lepton are accepted, whereas the presence of any additional lepton passing the loose identification requirements with $p_{\mathrm{T}}>10 \mathrm{GeV}$ vetoes the event. Moreover, the presence of a $\tau_{\mathrm{h}}$ candidate with $p_{\mathrm{T}}>20 \mathrm{GeV}$ and $|\eta|<2.3$ vetoes the event. In the dilepton category, we accept events with exactly two oppositely charged leptons (electrons or muons); the second lepton is required to have $p_{\mathrm{T}}>10 \mathrm{GeV}$ and pass looser identification criteria than the leading lepton. To reduce the $\mathrm{Z} / \gamma^{*}$ background, we reject events with two leptons of the same flavor and opposite charge with an invariant mass $m_{\ell \ell}$ less than 12 or between 76 and $106 \mathrm{GeV}$.

The final states examined in this paper include neutrinos from the $\mathrm{W}$ boson decays; events are therefore required to have $p_{\mathrm{T}}^{\text {miss }}>30 \mathrm{GeV}$. Additionally, in the single-lepton final state, events in which the $p_{\mathrm{T}}^{\text {miss }}$ is compatible with mismeasurement of electron or jet energy are rejected by requiring the azimuthal angle separation between the $p_{\mathrm{T}}^{\text {miss }}$ and any jet in the event to be $\Delta \phi>0.05$.

Tree-level signal production processes are characterized by having five (three) jets at

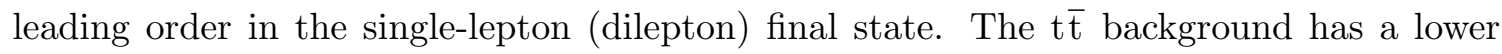
jet multiplicity in the corresponding regions, but additional jets may be produced through initial- and final-state radiation. Requiring a high multiplicity of reconstructed jets improves the discrimination of signal events from the background, while the regions depleted in signal processes constrain background estimates using data. Consequently, in the singlelepton and dilepton event regions, the presence of at least four and two jets, respectively, is 
required. The SM top quark pair production has final states similar to the charged Higgs boson signal production with fewer b quarks at tree level, while additional gluon splitting contaminates the high $\mathrm{b}$ jet multiplicity regions. Consequently, one or more of these jets is required to be b-tagged.

Events are categorized according to the total number of associated jets $N_{\text {jets }}$ and the b-tagged jet multiplicity $N_{\mathrm{b} \text { jets }}$, yielding a total of nine regions in the single-lepton final state and eight regions in the dilepton final state. In the single-lepton final state, the regions are: $(4 \mathrm{j} / 1 \mathrm{~b}),(4 \mathrm{j} / 2 \mathrm{~b}),(4 \mathrm{j} / \geq 3 \mathrm{~b}),(5 \mathrm{j} / 1 \mathrm{~b}),(5 \mathrm{j} / 2 \mathrm{~b}),(5 \mathrm{j} / \geq 3 \mathrm{~b}),(\geq 6 \mathrm{j} / 1 \mathrm{~b}),(\geq 6 \mathrm{j} / 2 \mathrm{~b})$, and $(\geq 6 \mathrm{j} / \geq 3 \mathrm{~b})$; while in the dilepton final states, where less hadronic activity is expected, the regions are: $(2 \mathrm{j} / 1 \mathrm{~b}),(2 \mathrm{j} / 2 \mathrm{~b}),(3 \mathrm{j} / 1 \mathrm{~b}),(3 \mathrm{j} / 2 \mathrm{~b}),(3 \mathrm{j} / 3 \mathrm{~b}),(\geq 4 \mathrm{j} / 1 \mathrm{~b}),(\geq 4 \mathrm{j} / 2 \mathrm{~b})$, and $(\geq 4 \mathrm{j} / \geq 3 \mathrm{~b})$. The resulting regions are characterized by different background compositions and signal purities, and are collectively labeled signal regions and used in the likelihood fit for signal extraction. We additionally define control regions which we use to correct from data the normalization of background samples; these regions are described in section 6 .

For a large $\mathrm{H}^{+}$mass range, the highest significance for both the single-lepton and dilepton final states is found in the regions having higher $N_{\text {jets }}$ and $N_{\text {b jets. The only }}$ exception are the $\mathrm{H}^{+}$signals with the mass around $200 \mathrm{GeV}$, where the low $N_{\text {jets }}$ and $N_{\text {b jets }}$ regions have higher sensitivity than the high multiplicity ones. Finally, events with two same-sign leptons are used to form control regions for the multijet background estimation.

A set of discriminant variables is selected to enhance the signal and background separation in each category and is summarized in table 1.

Kinematic and topological shapes have different discrimination power for the different mass hypotheses of the charged Higgs boson. Each discriminant variable is studied and included in an MVA classifier if it improves the discrimination, or otherwise discarded. For both single-lepton and dilepton regions, the $H_{\mathrm{T}}$ distribution, defined as the scalar sum of the $p_{\mathrm{T}}$ of the selected jets, is one of the most sensitive variables. Additionally, the largest $p_{\mathrm{T}}$ among the $\mathrm{b}$ jets, the $p_{\mathrm{T}}^{\text {miss }}$, the minimum invariant mass between the lepton and the b jets, the maximum $\Delta \eta$ between two b-tagged jets, the smallest $\Delta R$ separation of the $\mathrm{b}$ jets, and the $p_{\mathrm{T}}$-weighted average of the $\mathrm{b}$ tagging discriminator calculated using the non-b-tagged jets are used as input variables to the MVA discriminators. Information about the event topology is incorporated via event shape variables, such as the centrality which is defined as the ratio of the sum of the transverse momenta of all jets to their total energy, and the second Fox-Wolfram moment [96] calculated using all jets.

In the single-lepton final states, the following variables are also included: the invariant mass of the three jets with largest $p_{\mathrm{T}}$, the transverse mass of the system constituted by the lepton and the $p_{\mathrm{T}}^{\text {miss }}$, the angular separation between the lepton and the system constituted by the $\mathrm{b}$ jet pair with the smallest $\Delta R$ separation between the b jets, and the average separation between the $\mathrm{b}$ jet pairs.

The event selection for the dilepton final state takes advantage of the presence of the second lepton. The lepton with largest $p_{\mathrm{T}}$ (leading lepton) characterizes the decay of a Lorentz-boosted top quark that originates from the massive charged Higgs boson in the signal hypothesis. The following variables are also considered: the $\Delta R$ between the leading lepton and the leading b-tagged jet, the momentum of the leading lepton, the 


\begin{tabular}{|c|c|c|}
\hline \multirow{9}{*}{ 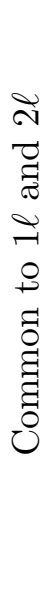 } & $H_{\mathrm{T}}$ & Scalar sum of the jet transverse momenta \\
\hline & $p_{\mathrm{Tb}}$ & Largest $p_{\mathrm{T}}$ among the b-tagged jets \\
\hline & $p_{\mathrm{T}}^{\mathrm{miss}}$ & Missing transverse momentum \\
\hline & $\min m(\ell, \mathrm{b})$ & Minimum invariant mass between the lepton and the b-tagged jet \\
\hline & $\max \Delta \eta(\mathrm{b}, \mathrm{b})$ & Maximum pseudorapidity separation between b-tagged jet pairs \\
\hline & $\min \Delta R(\mathrm{~b}, \mathrm{~b})$ & Minimum separation between b-tagged jet pairs \\
\hline & $p_{\mathrm{T}^{-}}\langle\mathrm{CSV}\rangle$ & $\begin{array}{l}p_{\mathrm{T}} \text { weighted average of the combined secondary vertex discrimi- } \\
\text { nator of the non-b-tagged jets }\end{array}$ \\
\hline & $F W_{2}$ & Second Fox-Wolfram moment \\
\hline & centrality & Ratio of the sum of the $p_{\mathrm{T}}$ and the total energy of all jets \\
\hline \multirow{4}{*}{$\stackrel{2}{2}$} & $m_{j j j}$ & $\begin{array}{l}\text { Invariant mass of the jet system composed by the first three jets } \\
\text { ranked in } p_{\mathrm{T}}\end{array}$ \\
\hline & $m_{\mathrm{T}}\left(\ell, \vec{p}_{\mathrm{T}}^{\mathrm{miss}}\right)$ & $\begin{array}{l}\text { Transverse mass of the system constituted by the lepton and the } \\
\vec{p}_{\mathrm{T}}^{\text {miss }}\end{array}$ \\
\hline & $\Delta R(\ell, \mathrm{bb})$ & $\begin{array}{l}\text { Distance between the b-tagged jet pair with the smallest } \Delta R \\
\text { separation and the lepton }\end{array}$ \\
\hline & $\langle\Delta R(\mathrm{~b}, \mathrm{~b})\rangle$ & Average separation between b-tagged jet pairs \\
\hline \multirow{7}{*}{ ๙ึ } & $N_{\text {jets }}$ & Number of selected jets \\
\hline & $N_{\text {b jets }}$ & Number of selected b-tagged jets \\
\hline & $\Delta R(\ell, \mathrm{b})$ & $\begin{array}{l}\text { Distance between the lepton and the b-tagged jet with largest } \\
\text { transverse momenta }\end{array}$ \\
\hline & $p_{\mathrm{T} \ell}$ & Largest $p_{\mathrm{T}}$ between the leptons \\
\hline & $\frac{p_{\mathrm{T} \ell 1}-p_{\mathrm{T} \ell 2}}{p_{\mathrm{T} \ell 1}+p_{\mathrm{T} \ell 2}}$ & Lepton $p_{\mathrm{T}}$ asymmetry \\
\hline & $m(\ell, \mathrm{b})$ & $\begin{array}{l}\text { Invariant mass of the lepton }+ \text { b-tagged jet system with the largest } \\
p_{\mathrm{T}} \text { (top quark candidate) }\end{array}$ \\
\hline & $m_{\mathrm{T}}^{\min }$ & $\begin{array}{l}\text { The smallest of the transverse masses constructed with the lead- } \\
\text { ing b-tagged jet and each of the two } \mathrm{W} \text { boson hypotheses: } \\
\min \left[m_{\mathrm{T}}\left(\mathrm{b}, p_{\mathrm{T} \ell 1}+\vec{p}_{\mathrm{T}}^{\text {miss }}\right), m_{\mathrm{T}}\left(\mathrm{b}, p_{\mathrm{T} \ell 2}+\vec{p}_{\mathrm{T}}^{\text {miss }}\right)\right]\end{array}$ \\
\hline
\end{tabular}

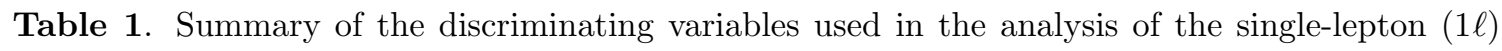
and dilepton $(2 \ell)$ final states.

lepton $p_{\mathrm{T}}$ asymmetry, the mass of the lepton+b-tagged jet system with the largest $p_{\mathrm{T}}$, and the smallest of the transverse masses constructed with the leading $b$ jet and each of the two $\mathrm{W}$ boson hypotheses, where the $\mathrm{W}$ bosons are reconstructed using the $\vec{p}_{\mathrm{T}}^{\text {miss }}$ and the lepton momenta.

Separate classifiers are constructed for the single-lepton and dilepton final states, using different technologies in order to fully exploit the different sets of features described above. 

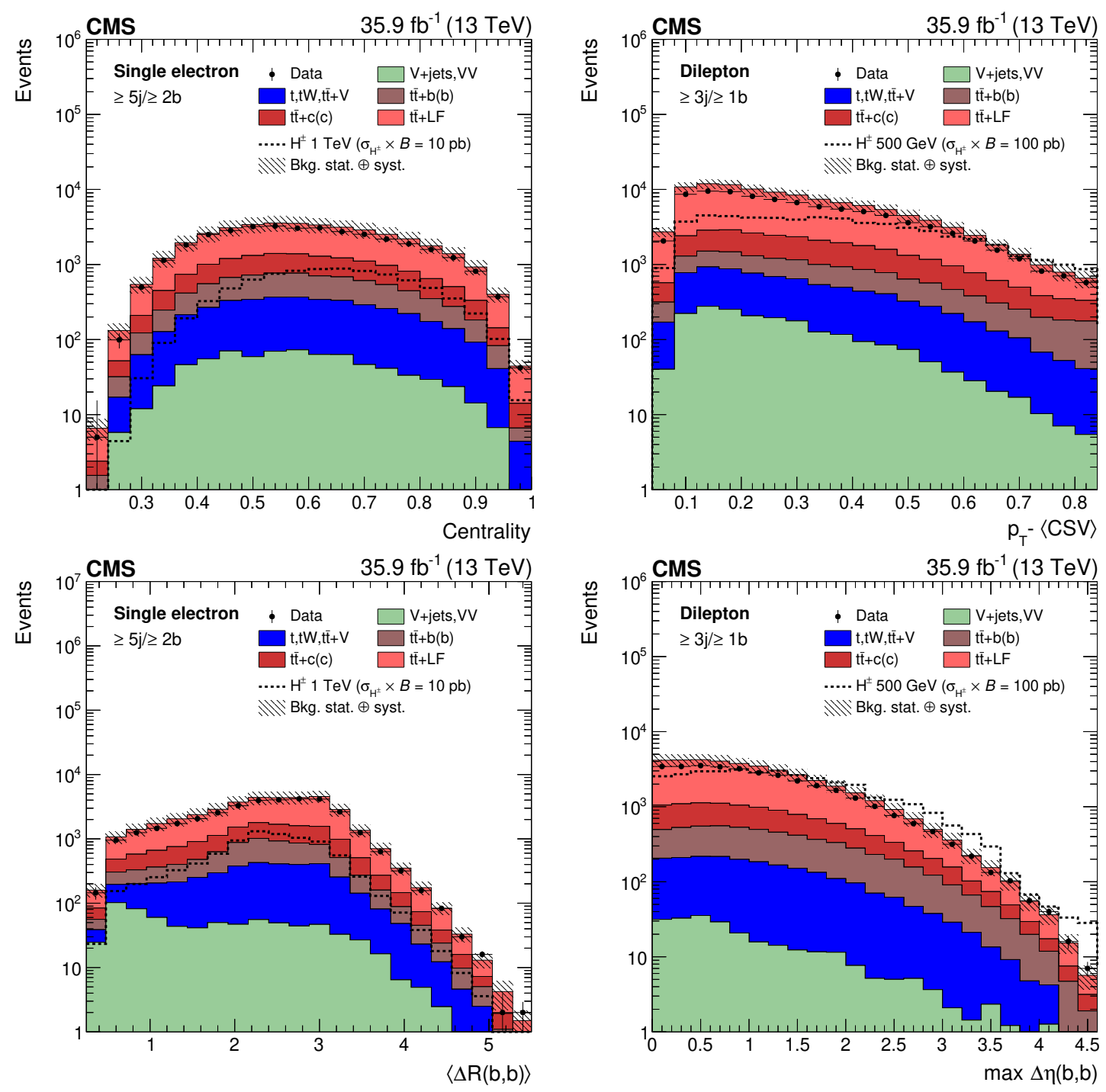

Figure 2. Representative input variables for the single-lepton $\geq 5 \mathrm{j} / \geq 2 \mathrm{~b}$ category (left) and for the dilepton $\geq 3 \mathrm{j} / \geq 1 \mathrm{~b}$ category (right) before the signal extraction fit. For the single-lepton final state, the centrality (top) and $\langle\Delta R(\mathrm{~b}, \mathrm{~b})\rangle$ (bottom) are shown; for the dilepton final state, the $p_{\mathrm{T}}-\langle\mathrm{CSV}\rangle$ (top) and $\max \Delta \eta(\mathrm{b}, \mathrm{b})$ (bottom) are shown. The black markers show the data. The solid histograms represent the background prediction for $t \bar{t}+\mathrm{LF}$ (light red), $t \bar{t}+\mathrm{c}(\mathrm{c})$ (dark red), $\mathrm{t} \overline{\mathrm{t}}+\mathrm{b}(\mathrm{b})$ (brown), single top quark and $\mathrm{t} \overline{\mathrm{t}}$ in association with extra bosons (blue), and $\mathrm{V}+$ jets and multiboson production (light green). The dashed line represents the yields for a charged Higgs boson with a mass of $1 \mathrm{TeV}(500 \mathrm{GeV})$ for the single lepton (dilepton) final state and a product of the cross section and branching fraction of 10 (100) pb for the single-lepton and dilepton final states, respectively. The hatched uncertainty bands include the total uncertainty before the signal extraction fit.

For each of the suitable discriminating variables, it has been verified that the simulation models data correctly. Figure 2 shows some of the most important input variables in exemplary signal-region subcategories for the single-lepton $(\geq 5 \mathrm{j} / \geq 2 \mathrm{~b})$ and dilepton final states $(\geq 3 \mathrm{j} / \geq 1 \mathrm{~b})$. 
For all the classifiers described below each signal and background sample is randomly divided into three equally populated parts; one third is used for training the classifiers, one third is used for testing the performance of the classifiers, and one third is used for evaluating the classifier in the context of the maximum-likelihood fit detailed in section 7 . The backgrounds are dominated by $t \bar{t}$ events, but all other SM contributions are also included in the training. Both in the single-lepton and the dilepton regions, the training process and possible sources of over- or under-training are verified by means of statistical tests.

A boosted decision tree (BDT) [97, 98] classifier is trained using the TMVA package [99] to discriminate between signal and background in the single-lepton regions. The dependence of the kinematic signature on $m_{\mathrm{H}^{ \pm}}$is accounted for by having a separate training for each $m_{\mathrm{H}^{ \pm}}$hypothesis. The training process is optimized by targeting a region enriched in signal events by requiring $N_{\text {jets }} \geq 5$ and $N_{\text {b jets }} \geq 2$ (training region). The binned output distribution of the BDT classifier is calculated in all the single-lepton subcategories corresponding to the training region plus the $(4 \mathrm{j} / \geq 3 \mathrm{~b})$ region and used in the maximum likelihood fit. In the other single-lepton subcategories, the inclusive event yields are used in the fit to infer additional information on the background normalization.

The dilepton final states exploit a novel technology based on deep neural network (DNN) classifiers [97], parametrized as a function of $m_{\mathrm{H}^{ \pm}}$[100]. The TENSORFLOW (v1.4.0) backend [101] and the KerAs (v2.1.1) frontend [102] are used to train the classifier. The parametrization of the signal events as a function of $m_{\mathrm{H}^{ \pm}}$enables a unique training for each signal mass hypothesis. The training process is optimized in the region enriched in signal events by requiring $N_{\text {jets }} \geq 3$ and $N_{\text {b jets }} \geq 1$. The jet and b-tagged jet multiplicities are used in extending the training parametrization to capture the characteristics of the signal and background processes in the different regions. In the regions characterized by a single $b$ jet we use the non-tagged jet with the highest value of the $b$ tagging discriminator as the second $b$ jet for the purpose of computing the input variables. The binned DNN output is used in the maximum likelihood fit in all the dilepton subcategories to further enhance the separation between the different background processes.

The bin size for the MVA output in each of the subcategories of the analysis is chosen with a variable binning strategy such that the statistical uncertainty in signal and background event yields separately is less than $20 \%$ in each bin. In order to avoid possible biases in the binning strategy induced by the statistical fluctuations in the simulated samples, the bin boundaries are defined based on the events used for the MVA training.

\section{Background estimation and systematic uncertainties}

The leptonic decay of one or two of the $\mathrm{W}$ bosons in the te process represents the main background of the analysis for both the single-lepton and dilepton final states. The t $\bar{t}$ production, as discussed in section 3 , is separated into $t \bar{t}+L F, t \bar{t}+b(b)$, and $t \bar{t}+c(c)$ processes. The last two processes are commonly referred to as $t \bar{t}+$ heavy flavor (HF). The categorization strategy described in section 5 populates the low b jet multiplicity regions with the $t \bar{t}+L F$ processes, while the regions enriched with the signal are characterized 
by a larger contribution from the $t \bar{t}+\mathrm{HF}$ processes. Smaller background contributions arise from single top quark production, vector boson production in association with jets, multiboson production processes, $t \bar{t}$ production in association with electroweak bosons $(\mathrm{W}$, $\mathrm{Z}, \gamma, \mathrm{H})$, and $\mathrm{t} \overline{\mathrm{t}} \mathrm{t} \overline{\mathrm{t}}$ production.

Different sources of experimental and theoretical uncertainties are modelled as nuisance parameters in the fit and they are allowed to change the event yield, the migration of events among regions, and the distribution of the MVA output in each category [103]. Uncertainties that purely affect the yield within a category (rate uncertainties) are modelled via a nuisance parameter with a log-normal probability density function, while changes in shapes (shape uncertainties) are performed using a polynomial interpolation with a Gaussian constraint, and they can also change the event yields. All the sources of systematic uncertainty applied to the analysis are discussed below.

The uncertainty in the integrated luminosity measurement of the 2016 dataset amounts to $2.5 \%$ [104]. The uncertainty in the evaluation of the pileup in simulation is accounted for by varying the total inelastic pp cross section by $\pm 5 \%$ and propagating the effect of the variation to the final yields. The difference between the nominal and the altered distributions is taken as the uncertainty and treated as a shape variation in the fit. Both the integrated luminosity and the pileup uncertainties are separately treated as fully correlated among all processes.

Each reconstructed jet is corrected via calibration factors in order to account for the response of the detectors, with dependencies on the geometry, the pileup conditions, and the kinematic properties of the jet [89]. The uncertainties in the jet energy scale and resolution are propagated by varying the jet momenta and, consequently, the missing transverse momentum. The events are reanalyzed in order to extract the appropriate rate and shape variations for the final distributions. An additional uncertainty accounts for the effect of the unclustered energy on $p_{\mathrm{T}}^{\mathrm{miss}}$. Each of these uncertainties is treated as fully correlated among all processes.

The $\mathrm{b}$ tagging and mistagging uncertainties are obtained by varying the corresponding per-jet correction factors within their uncertainties [91]. The mistag efficiency uncertainties for jets originating from light partons $(\mathrm{u}, \mathrm{d}, \mathrm{s}$, and $\mathrm{g})$ are considered to be uncorrelated with the $b$ tagging efficiency uncertainties, while the c quark jet mistag rate uncertainties are varied simultaneously with the $\mathrm{b}$ tagging efficiencies. The $\mathrm{b}$ tagging and mistagging efficiency uncertainties are conservatively doubled whenever they are extrapolated outside the $p_{\mathrm{T}} / \eta$ range over which the correction factors were derived. Different sources of uncertainties are varied as independent nuisance parameters. The portion of the $\mathrm{b}$ tagging efficiency uncertainty that is correlated with the jet energy scale is evaluated within the overall jet energy scale uncertainty by shifting the b tagging scale factors in the same direction as the jet energy scale shift; the procedure reflects the correlation in the derivation of the correction factors.

The uncertainties in the lepton selection efficiency correction factors due to trigger, identification, and isolation efficiencies are applied depending on the lepton $p_{\mathrm{T}}$ and $\eta$. The propagation of the correction factors on the shape of the MVA output impacts only the overall normalization. The squared sum of the variations due to the identification, isolation, 
and trigger efficiencies is therefore included as a single rate uncertainty amounting to 3 (4)\% for electrons (muons), treated as correlated among all the final regions.

Small discrepancies between data and simulation are observed in control regions enriched in processes involving a vector boson with additional jets. The $\mathrm{Z} / \gamma^{*}$ and $\mathrm{W}+$ jets $H_{\mathrm{T}}$ distributions are matched to data using corrections derived in a region close to the mass of the $\mathrm{Z}$ boson and in the zero $\mathrm{b}$ jet control region, respectively. The uncertainties in the derivation of correction factors for the $\mathrm{Z} / \gamma^{*}$ and $\mathrm{W}+$ jets processes in the $H_{\mathrm{T}}$ distribution are accounted for in the final results. They are assumed to be uncorrelated between the two processes and correlated among the analysis regions.

The QCD multijet production is a minor background to the analysis, amounting to about $1 \%$ of the total background across all the signal regions, and is therefore ignored in the fit after the verification of the simulated prediction. For the single-lepton regions, the simulation has been checked in an orthogonal set of events requiring that the $p_{\mathrm{T}}^{\text {miss }}$ is aligned with the jets, while for the dilepton regions, the QCD multijet production is verified in the same-sign dilepton control regions for each category defined by $N_{\text {jets }}$ and $N_{\text {b jets }}$.

Theoretical uncertainties related to the PDFs are applied as rate uncertainties to the simulated background samples and account for both the acceptance and the cross section mismodelling [105]. Uncertainties from factorization and renormalization scales in the inclusive cross sections are considered independently for each process for which they are non negligible. They are estimated by varying each scale independently from the others by factors of 0.5 and 2 with respect to the default values.

For the simulated samples involving a top quark, an additional uncertainty in the cross sections due to the choice of the top quark mass is considered by varying the top quark mass by $\pm 1.0 \mathrm{GeV}$ around the nominal value of $172.5 \mathrm{GeV}$.

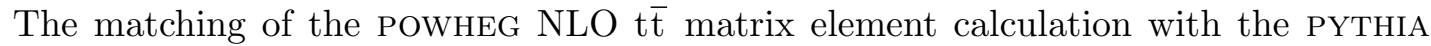
parton shower (PS) is varied by shifting the parameter $h_{\mathrm{damp}}=1.58_{-0.59}^{+0.66} m_{\mathrm{t}}[106]$ within the uncertainties. The damping factor $h_{\text {damp }}$ is used to limit the resummation of higher-order effects by the Sudakov form factor to below a given $p_{\mathrm{T}}$ scale [106].

An additional source of uncertainty arises from the modeling of additional jets by the event generator in top quark pair production. This uncertainty is estimated in each bin of jet and $b$ jet multiplicity, based on the simulated $t \bar{t}$ samples which are enriched or depleted in initial- and final-state radiation. The initial-state radiation PS scale is multiplied by factors of 2 and 0.5 in dedicated simulated samples, whereas the final-state radiation PS scale is scaled up by $\sqrt{2}$ and down by $1 / \sqrt{2}[63,106]$. For each PS scale and $h_{\text {damp }}$ perturbation, the uncertainty is evaluated as the relative deviation with respect to the nominal event rates. A nuisance parameter is added for each category defined by $N_{\text {jets }}$ and $N_{\text {b jets }}$ and considered uncorrelated among regions with different $N_{\text {jets }}$ and also uncorrelated between the single-lepton and dilepton final states.

The normalization of the $\mathrm{t} \overline{\mathrm{t}}+\mathrm{HF}$ processes, as determined by theoretical calculations [107] and experimental measurements, is affected by an uncertainty of $50 \%$ that is applied as a rate uncertainty, in addition to the other t $\bar{t}$ cross section uncertainties described above. This procedure allows the signal-depleted regions to determine the overall 


\begin{tabular}{|lccccccc|}
\hline \multicolumn{1}{|c}{ Source of uncertainty } & Shape & $\mathrm{H}^{ \pm}$ & $\mathrm{t} \overline{\mathrm{t}}+\mathrm{LF}$ & $\mathrm{t} \overline{\mathrm{t}}+\mathrm{c}(\mathrm{c})$ & $\mathrm{t} \overline{\mathrm{t}}+\mathrm{b}(\mathrm{b})$ & $\mathrm{t}, \mathrm{tW}, \mathrm{t} \overline{\mathrm{t}}+\mathrm{X}$ & $\mathrm{V}+\mathrm{jets}$ \\
\hline Integrated luminosity & & 2.5 & 2.5 & 2.5 & 2.5 & 2.5 & 2.5 \\
Pileup & $\checkmark$ & 0.4 & 0.2 & 0.2 & 0.2 & 0.2 & 1.8 \\
Jet energy scale and resolution & $\checkmark$ & 2.8 & 3.9 & 3.3 & 3.0 & 3.9 & 5.3 \\
$\mathrm{~b}$ jet identification & $\checkmark$ & 4.6 & 3.1 & 4.1 & 4.6 & 3.0 & 11.6 \\
Lepton selection efficiency & & 3.4 & 3.1 & 3.3 & 3.3 & 3.3 & 3.7 \\
Unclustered pmiss energy & & 2.0 & 2.0 & 2.0 & 2.0 & 2.0 & 2.0 \\
Acceptance (scales, PDF) & $\checkmark$ & 9.8 & 9.0 & 11.4 & 12.0 & 3.3 & 11.2 \\
Cross section (scales, PDF) & & - & 5.5 & 5.5 & 5.5 & 4.0 & 4.1 \\
Top quark mass & & - & 2.7 & 2.7 & 2.7 & 2.2 & - \\
$\mathrm{t} \overline{\mathrm{t}}$ parton showering & & - & 6.4 & 10.6 & 9.5 & - & - \\
$\mathrm{t} \overline{\mathrm{t}}+\mathrm{HF}$ normalization & & - & - & 50.0 & 50.0 & - & - \\
\hline
\end{tabular}

Table 2. Effects of the systematic uncertainties as the variation (in percent) of the event yields prior to the fit to data, summed over all final states and regions. The column Shape reports whether a given uncertainty is considered a shape uncertainty or a rate uncertainty.

normalization factor, which includes the production cross section, detector acceptance, and reconstruction efficiencies.

The limited size of the background and signal simulated samples results in statistical fluctuations of the nominal yield prediction. The content of each bin of each final discriminant distribution is varied by its statistical uncertainty. The Barlow-Beeston lite approach $[108,109]$ is applied by assigning, for each bin, the combined statistical uncertainty of all simulated samples to the process dominating the background yield in that bin. Since all bins are statistically independent, each variation is treated as uncorrelated with any other variation.

A summary of the effects of the systematic uncertainties on the event yields, summed over all final states and regions, is provided prior to the fit to data in table 2 .

\section{Results}

The statistical interpretation is based on a simultaneous fit of the MVA output discriminators and event yields in the different signal regions described in section 5 . The parameter of interest reflecting the signal normalization $\sigma_{\mathrm{H}^{ \pm}} \mathcal{B}\left(\mathrm{H}^{ \pm} \rightarrow \mathrm{tb}\right)=\sigma\left(\mathrm{pp} \rightarrow \mathrm{H}^{+} \overline{\mathrm{t}} \mathrm{b}+\mathrm{pp} \rightarrow\right.$ $\left.\mathrm{H}^{+} \overline{\mathrm{t}}\right) \mathcal{B}\left(\mathrm{H}^{+} \rightarrow \mathrm{t} \overline{\mathrm{b}}\right)+\sigma\left(\mathrm{pp} \rightarrow \mathrm{H}^{-} \mathrm{t} \overline{\mathrm{b}}+\mathrm{pp} \rightarrow \mathrm{H}^{-} \mathrm{t}\right) \mathcal{B}\left(\mathrm{H}^{-} \rightarrow \overline{\mathrm{t} b}\right)$ and the nuisance parameters specified in section 6 are encoded in the negative log-likelihood function and profiled in the minimization process. The log-likelihood ratio is used as test statistic to assess the agreement of data with the background-only hypothesis or the presence of the signal and the asymptotic approximation is used in the statistical analysis $[103,110]$. The statistical method used to report the results is the $\mathrm{CL}_{\mathrm{s}}$ modified frequentist criterion [111, 112].

Figure 3 shows the event yields in the subcategories of the analysis after a backgroundonly fit to data. In the regions where the shape of the MVA classifier output is used, the yields are obtained by integrating the distribution and the correlations across the bins 

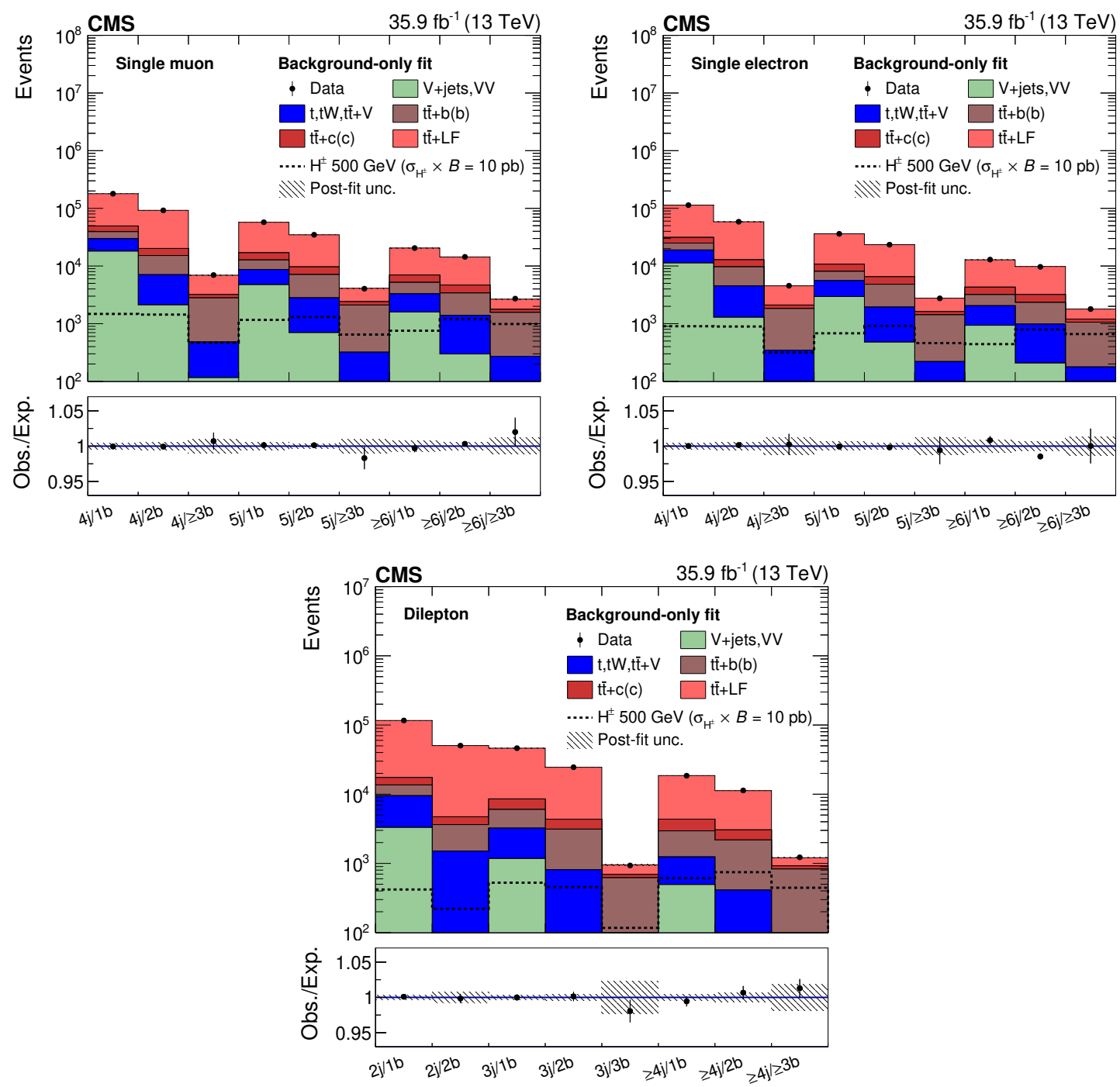

Figure 3. Summary of event yields in each analysis category for single-muon (top left), singleelectron (top right), and dilepton (bottom) final states. The yields observed in data (black markers) are overlaid. The solid histograms represent the background prediction for $t \bar{t}+\mathrm{LF}$ (light red), $\mathrm{t} \overline{\mathrm{t}}+\mathrm{c}(\mathrm{c})$ (dark red), $\mathrm{t} \overline{\mathrm{t}}+\mathrm{b}(\mathrm{b})$ (brown), single top quark and $\mathrm{t} \overline{\mathrm{t}}$ in association with extra bosons (blue), and $\mathrm{V}+$ jets and multiboson production (light green). The dashed line represents the yields for a charged Higgs boson with a mass of $500 \mathrm{GeV}$ and a product of the cross section and the branching fraction of $10 \mathrm{pb}$. The lower panel shows the ratio of data to the SM expectation after the background-only fit to the data and the hatched uncertainty bands include the total uncertainty.

are accounted for in the quoted uncertainties. The contribution of a hypothetical charged Higgs boson with a mass of $500 \mathrm{GeV}$ and $\sigma_{\mathrm{H}^{ \pm}} \mathcal{B}\left(\mathrm{H}^{ \pm} \rightarrow \mathrm{tb}\right)=10 \mathrm{pb}$ is also displayed. In the same configuration, figure 4 shows the MVA (BDT and DNN) outputs in exemplary signal-region subcategories for the single-lepton $(5 \mathrm{j} / \geq 3 \mathrm{~b})$ and dilepton $(3 \mathrm{j} / 3 \mathrm{~b})$ final states.

The data agree with the background distributions and no significant excess is observed. Exclusion limits are set at $95 \%$ confidence level (CL) on $\sigma_{\mathrm{H}^{ \pm}} \mathcal{B}\left(\mathrm{H}^{ \pm} \rightarrow\right.$ tb) for $m_{\mathrm{H}^{ \pm}}$ 

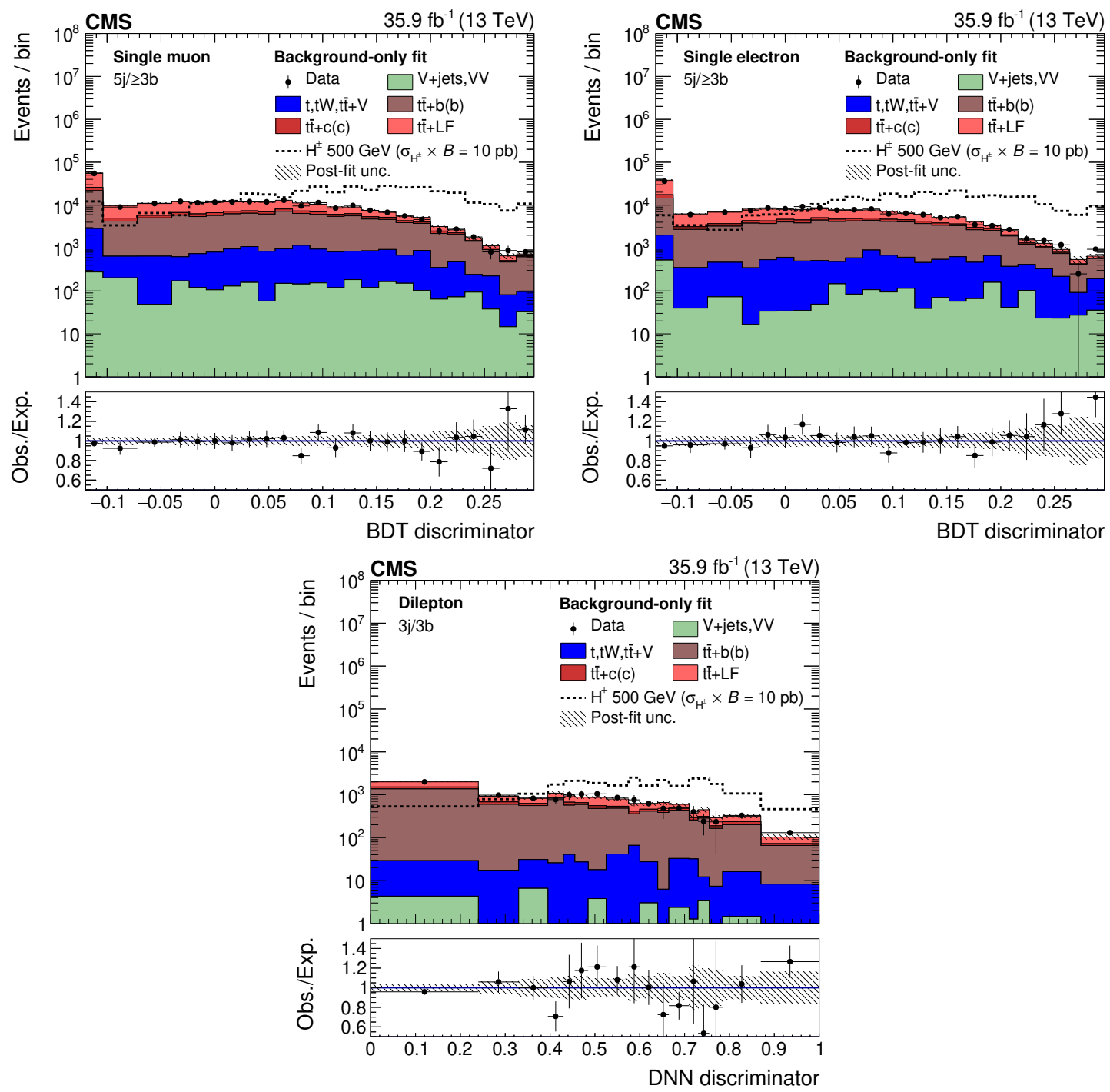

Figure 4. Distributions of the MVA outputs of the data and the SM expectation after the background-only fit to the data for the single-muon $5 \mathrm{j} / \geq 3 \mathrm{~b}$ category (top left), for the singleelectron $5 \mathrm{j} / \geq 3 \mathrm{~b}$ category (top right), and for the dilepton $3 \mathrm{j} / 3 \mathrm{~b}$ category (bottom). The black markers show the data. The solid histograms represent the background prediction for $\mathrm{t} \overline{\mathrm{t}}+\mathrm{LF}$ (light red), $t \bar{t}+c(c)$ (dark red), $t \bar{t}+b(b)$ (brown), single top quark and $t \bar{t}$ in association with extra bosons (blue), and $\mathrm{V}+$ jets and multiboson production (light green). The dashed line represents the yields for a charged Higgs boson with a mass of $500 \mathrm{GeV}$ and a product of the cross section and branching fraction of $10 \mathrm{pb}$. The lower panel shows the ratio of data to the SM expectation after the background-only fit to the data and the hatched uncertainty bands include the total uncertainty.

hypotheses between 200 and $3000 \mathrm{GeV}$. The observed (expected) upper limits with singlelepton and dilepton final states combined are shown in figure 5 (left) and listed in table 3. The single-lepton and dilepton regions have comparable sensitivity in the low-mass regime $(\approx 200 \mathrm{GeV})$ while the single-lepton regions become increasingly dominant at higher values of the mass hypothesis; figure 5 (right) details the contributions of the single-lepton and dilepton regions. Using the MVA classifier instead of the $H_{\mathrm{T}^{-}}$based approach of the pre- 

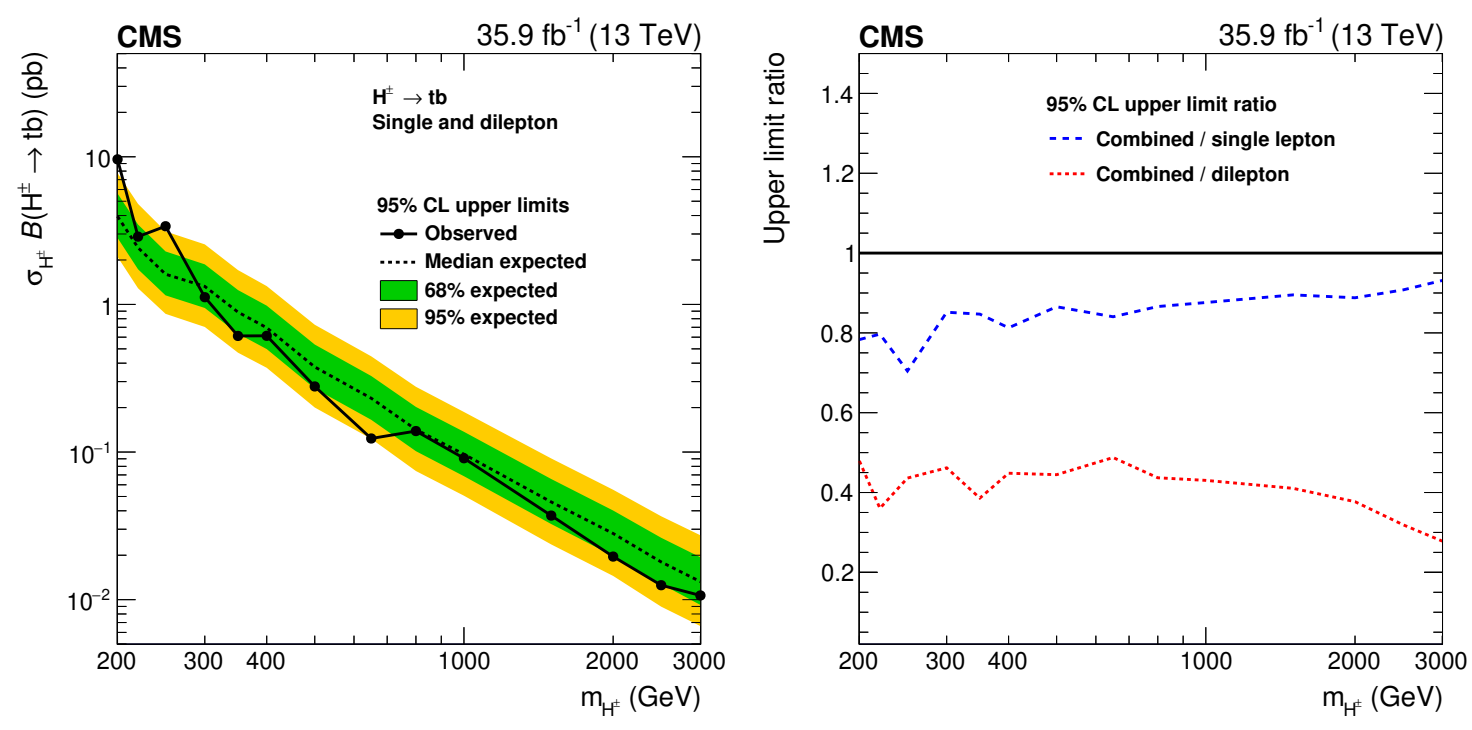

Figure 5. The upper limit at 95\% CL on $\sigma_{\mathrm{H}^{ \pm}} \mathcal{B}\left(\mathrm{H}^{ \pm} \rightarrow \mathrm{tb}\right)$ with single-lepton and dilepton final states combined (left). The solid black markers describe the observed upper limits, while the dashed line corresponds to the expectations from the background. The green (yellow) band represents one (two) standard deviations from the expected median. The contribution of the single-lepton and dilepton regions to the combined limit is also represented, expressed as a ratio (right).

vious publication [32] yields an improvement of $20-40 \%$ in the expected limits, depending on the signal mass.

The model-dependent upper limits are obtained by comparing the observed limits with the theoretical predictions. The MSSM $m_{\mathrm{h}}^{\bmod -}$ benchmark scenario [17] is designed to give a mass of approximately $125 \mathrm{GeV}$ for the light CP-even 2HDM Higgs boson over a wide region of the parameter space. The $M_{\mathrm{h}}^{125}(\tilde{\chi})$ scenario [113] is characterized by small gaugino and Higgs/higgsino superpotential masses which are also close to each other; this results in a significant mixing parameter between higgsinos and gauginos and in a compressed electroweakino mass spectrum. The phenomenology of the $M_{\mathrm{h}}^{125}(\tilde{\chi})$ scenario resembles therefore the Type-II 2HDM with MSSM-inspired Higgs couplings compatible with $m_{\mathrm{h}} \approx 125 \mathrm{GeV}$ for large masses of the pseudoscalar boson, A. Figure 6 shows the excluded parameter space in the MSSM $m_{\mathrm{h}}^{\bmod -}$ and $M_{\mathrm{h}}^{125}(\tilde{\chi})$ scenarios. In both models, the observed exclusion of high values of $\tan \beta$ is in the range 40-60 in the $m_{\mathrm{H}^{ \pm}}$range of 200-700 GeV; for low values of $\tan \beta$ the values $0.4-1.5$ are excluded in the $m_{\mathrm{H}^{ \pm}}$range of $200 \mathrm{GeV}-1.5 \mathrm{TeV}$ in the context of $m_{\mathrm{h}}^{\bmod -}$ scenario while the values $0.6-1.5$ are excluded in the $m_{\mathrm{H}^{ \pm}}$range of $200 \mathrm{GeV}-1 \mathrm{TeV}$ for the $M_{\mathrm{h}}^{125}(\tilde{\chi})$ scenario.

\section{Summary}

A search is presented for a charged Higgs boson decaying into a top-bottom quark-antiquark pair when produced in association with a top quark or a top and a bottom quark. The analyzed proton-proton collision data, collected at $\sqrt{s}=13 \mathrm{TeV}$ with the CMS detector at 


\begin{tabular}{|ccccccc|}
\hline \multirow{2}{*}{$m_{\mathrm{H}^{ \pm}}(\mathrm{GeV})$} & \multicolumn{5}{c|}{ Expected limits $(\mathrm{pb})$} & \multirow{2}{*}{ Observed limits $(\mathrm{pb})$} \\
\cline { 2 - 6 } 200 & -2 s.d. & -1 s.d. & median expected & +1 s.d. & +2 s.d. & \\
220 & 2.1 & 2.8 & 4.0 & 5.6 & 7.8 & 9.6 \\
250 & 1.3 & 1.7 & 2.4 & 3.5 & 4.8 & 2.9 \\
300 & 0.9 & 1.2 & 1.6 & 2.3 & 3.1 & 3.4 \\
350 & 0.7 & 0.9 & 1.3 & 1.9 & 2.6 & 1.1 \\
400 & 0.47 & 0.63 & 0.89 & 1.25 & 1.71 & 0.61 \\
500 & 0.37 & 0.50 & 0.70 & 0.98 & 1.33 & 0.61 \\
650 & 0.20 & 0.27 & 0.38 & 0.53 & 0.73 & 0.28 \\
800 & 0.12 & 0.17 & 0.23 & 0.33 & 0.45 & 0.12 \\
1000 & 0.07 & 0.10 & 0.14 & 0.20 & 0.28 & 0.14 \\
1500 & 0.051 & 0.069 & 0.097 & 0.137 & 0.187 & 0.091 \\
2000 & 0.024 & 0.033 & 0.046 & 0.066 & 0.090 & 0.037 \\
2500 & 0.015 & 0.020 & 0.028 & 0.040 & 0.056 & 0.020 \\
3000 & 0.009 & 0.013 & 0.018 & 0.026 & 0.037 & 0.013 \\
& 0.007 & 0.009 & 0.013 & 0.019 & 0.027 & 0.011 \\
\hline
\end{tabular}

Table 3. The upper limits at $95 \% \mathrm{CL}$ on the $\sigma_{\mathrm{H}^{ \pm}} \mathcal{B}\left(\mathrm{H}^{ \pm} \rightarrow\right.$ tb) with the single-lepton and dilepton final states combined. The one (two) standard deviations (s.d.) from the expected median are also reported.
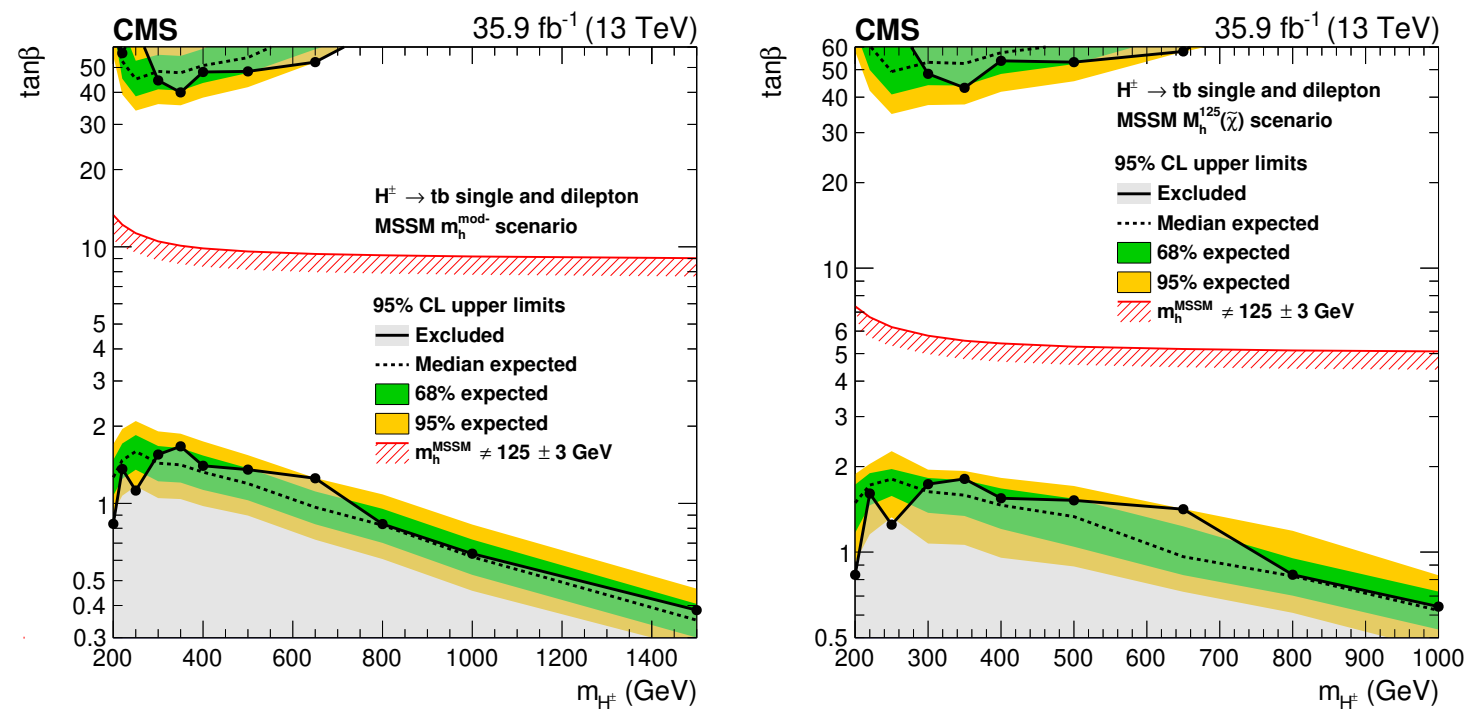

Figure 6. Excluded parameter space regions in the $m_{\mathrm{h}}^{\bmod -}$ scenario (left) and in the $M_{\mathrm{h}}^{125}(\tilde{\chi})$ scenario (right). The grey area delimited by the solid black line and markers represents the observed excluded region. The dashed black line and the green (yellow) regions represent the median expected exclusion regions and one (two) standard deviations from the expected median, respectively. The region below the red line is excluded assuming that the observed neutral Higgs boson is the light CP-even 2HDM Higgs boson with a mass of $125 \pm 3 \mathrm{GeV}$, where the uncertainty is the theoretical uncertainty in the mass calculation. 
the LHC, correspond to an integrated luminosity of $35.9 \mathrm{fb}^{-1}$. The search uses events with a single isolated electron or muon or an opposite-sign electron or muon pair. Events are categorized according to the jet multiplicity and the number of jets identified as containing a b-hadron decay. Multivariate techniques are used to discriminate between signal and background events, the latter being dominated by $t \bar{t}$ production. Results are presented for a charged Higgs boson with a mass larger than the top quark mass. 95\% confidence level upper limits of $9.6-0.01 \mathrm{pb}$ are set on the product of the charged Higgs boson production cross section and the branching fraction into a top-bottom quark-antiquark pair, $\sigma_{\mathrm{H}^{ \pm}} \mathcal{B}\left(\mathrm{H}^{ \pm} \rightarrow \mathrm{tb}\right)=\sigma\left(\mathrm{pp} \rightarrow \mathrm{H}^{+} \overline{\mathrm{tb}}+\mathrm{pp} \rightarrow \mathrm{H}^{+} \overline{\mathrm{t}}\right) \mathcal{B}\left(\mathrm{H}^{+} \rightarrow \mathrm{t} \overline{\mathrm{b}}\right)+\sigma\left(\mathrm{pp} \rightarrow \mathrm{H}^{-} \mathrm{t} \overline{\mathrm{b}}+\mathrm{pp} \rightarrow\right.$ $\left.\mathrm{H}^{-} \mathrm{t}\right) \mathcal{B}\left(\mathrm{H}^{-} \rightarrow \overline{\mathrm{t}} \mathrm{b}\right)$, in the mass range from $200 \mathrm{GeV}$ to $3 \mathrm{TeV}$, representing an improvement over previous results [32-34] by a factor of about 2-7 in the given mass range. Exclusion regions in the parameter space of the minimal supersymmetric standard model $m_{\mathrm{h}}^{\text {mod- }}$ and $M_{\mathrm{h}}^{125}(\tilde{\chi})$ benchmark scenarios are presented.

\section{Acknowledgments}

We congratulate our colleagues in the CERN accelerator departments for the excellent performance of the LHC and thank the technical and administrative staffs at CERN and at other CMS institutes for their contributions to the success of the CMS effort. In addition, we gratefully acknowledge the computing centers and personnel of the Worldwide LHC Computing Grid for delivering so effectively the computing infrastructure essential to our analyses. Finally, we acknowledge the enduring support for the construction and operation of the LHC and the CMS detector provided by the following funding agencies: BMBWF and FWF (Austria); FNRS and FWO (Belgium); CNPq, CAPES, FAPERJ, FAPERGS, and FAPESP (Brazil); MES (Bulgaria); CERN; CAS, MoST, and NSFC (China); COLCIENCIAS (Colombia); MSES and CSF (Croatia); RPF (Cyprus); SENESCYT (Ecuador); MoER, ERC IUT, PUT and ERDF (Estonia); Academy of Finland, MEC, and HIP (Finland); CEA and CNRS/IN2P3 (France); BMBF, DFG, and HGF (Germany); GSRT (Greece); NKFIA (Hungary); DAE and DST (India); IPM (Iran); SFI (Ireland); INFN (Italy); MSIP and NRF (Republic of Korea); MES (Latvia); LAS (Lithuania); MOE and UM (Malaysia); BUAP, CINVESTAV, CONACYT, LNS, SEP, and UASLP-FAI (Mexico); MOS (Montenegro); MBIE (New Zealand); PAEC (Pakistan); MSHE and NSC (Poland); FCT (Portugal); JINR (Dubna); MON, RosAtom, RAS, RFBR, and NRC KI (Russia); MESTD (Serbia); SEIDI, CPAN, PCTI, and FEDER (Spain); MOSTR (Sri Lanka); Swiss Funding Agencies (Switzerland); MST (Taipei); ThEPCenter, IPST, STAR, and NSTDA (Thailand); TUBITAK and TAEK (Turkey); NASU and SFFR (Ukraine); STFC (United Kingdom); DOE and NSF (U.S.A.).

Individuals have received support from the Marie-Curie program and the European Research Council and Horizon 2020 Grant, contract Nos. 675440, 752730, and 765710 (European Union); the Leventis Foundation; the A.P. Sloan Foundation; the Alexander von Humboldt Foundation; the Belgian Federal Science Policy Office; the Fonds pour la Formation à la Recherche dans l'Industrie et dans l'Agriculture (FRIA-Belgium); the Agentschap voor Innovatie door Wetenschap en Technologie (IWT-Belgium); the F.R.S.-FNRS and 
FWO (Belgium) under the "Excellence of Science — EOS" — be.h project n. 30820817; the Beijing Municipal Science \& Technology Commission, No. Z181100004218003; the Ministry of Education, Youth and Sports (MEYS) of the Czech Republic; the Lendület ("Momentum") Program and the János Bolyai Research Scholarship of the Hungarian Academy of Sciences, the New National Excellence Program ÚNKP, the NKFIA research grants 123842, 123959, 124845, 124850, 125105, 128713, 128786, and 129058 (Hungary); the Council of Science and Industrial Research, India; the HOMING PLUS program of the Foundation for Polish Science, cofinanced from European Union, Regional Development Fund, the Mobility Plus program of the Ministry of Science and Higher Education, the National Science Center (Poland), contracts Harmonia 2014/14/M/ST2/00428, Opus 2014/13/B/ST2/02543, 2014/15/B/ST2/03998, and 2015/19/B/ST2/02861, Sonatabis 2012/07/E/ST2/01406; the National Priorities Research Program by Qatar National Research Fund; the Ministry of Science and Education, grant no. 3.2989.2017 (Russia); the Programa Estatal de Fomento de la Investigación Científica y Técnica de Excelencia María de Maeztu, grant MDM-2015-0509 and the Programa Severo Ochoa del Principado de Asturias; the Thalis and Aristeia programs cofinanced by EU-ESF and the Greek NSRF; the Rachadapisek Sompot Fund for Postdoctoral Fellowship, Chulalongkorn University and the Chulalongkorn Academic into Its 2nd Century Project Advancement Project (Thailand); the Welch Foundation, contract C-1845; and the Weston Havens Foundation (U.S.A.).

Open Access. This article is distributed under the terms of the Creative Commons Attribution License (CC-BY 4.0), which permits any use, distribution and reproduction in any medium, provided the original author(s) and source are credited.

\section{References}

[1] ATLAS collaboration, Observation of a new particle in the search for the Standard Model Higgs boson with the ATLAS detector at the LHC, Phys. Lett. B 716 (2012) 1 [arXiv: 1207.7214] [INSPIRE].

[2] CMS collaboration, Observation of a New Boson at a Mass of 125 GeV with the CMS Experiment at the LHC, Phys. Lett. B 716 (2012) 30 [arXiv:1207.7235] [InSPIRE].

[3] CMS collaboration, Observation of a New Boson with Mass Near 125 GeV in pp Collisions at $\sqrt{s}=7$ and $8 \mathrm{TeV}$, JHEP 06 (2013) 081 [arXiv: 1303.4571] [INSPIRE].

[4] ATLAS and CMS collaborations, Combined Measurement of the Higgs Boson Mass in pp Collisions at $\sqrt{s}=7$ and 8 TeV with the ATLAS and CMS Experiments, Phys. Rev. Lett. 114 (2015) 191803 [arXiv:1503.07589] [INSPIRE].

[5] CMS collaboration, Measurements of properties of the Higgs boson decaying into the four-lepton final state in pp collisions at $\sqrt{s}=13$ TeV, JHEP 11 (2017) 047 [arXiv: 1706.09936] [INSPIRE].

[6] J.F. Gunion and H.E. Haber, The CP conserving two Higgs doublet model: The Approach to the decoupling limit, Phys. Rev. D 67 (2003) 075019 [hep-ph/0207010] [INSPIRE].

[7] A.G. Akeroyd et al., Prospects for charged Higgs searches at the LHC, Eur. Phys. J. C 77 (2017) 276 [arXiv:1607.01320] [INSPIRE]. 
[8] G.C. Branco, P.M. Ferreira, L. Lavoura, M.N. Rebelo, M. Sher and J.P. Silva, Theory and phenomenology of two-Higgs-doublet models, Phys. Rept. 516 (2012) 1 [arXiv:1106.0034] [INSPIRE].

[9] E. Gildener and S. Weinberg, Symmetry Breaking and Scalar Bosons, Phys. Rev. D 13 (1976) 3333 [INSPIRE].

[10] P. Fayet, Supergauge Invariant Extension of the Higgs Mechanism and a Model for the electron and Its Neutrino, Nucl. Phys. B 90 (1975) 104 [inSPIRE].

[11] P. Fayet, Supersymmetry and Weak, Electromagnetic and Strong Interactions, Phys. Lett. B 64 (1976) 159 [INSPIRE].

[12] P. Fayet, Spontaneously Broken Supersymmetric Theories of Weak, Electromagnetic and Strong Interactions, Phys. Lett. B 69 (1977) 489 [INSPIRE].

[13] S. Dimopoulos and H. Georgi, Softly Broken Supersymmetry and SU(5), Nucl. Phys. B 193 (1981) 150 [INSPIRE].

[14] N. Sakai, Naturalness in Supersymmetric Guts, Z. Phys. C 11 (1981) 153 [InSPIRE].

[15] K. Inoue, A. Kakuto, H. Komatsu and S. Takeshita, Low-Energy Parameters and Particle Masses in a Supersymmetric Grand Unified Model, Prog. Theor. Phys. 67 (1982) 1889 [INSPIRE].

[16] A. Djouadi, The Anatomy of electro-weak symmetry breaking. II. The Higgs bosons in the minimal supersymmetric model, Phys. Rept. 459 (2008) 1 [hep-ph/0503173] [INSPIRE].

[17] M. Carena, S. Heinemeyer, O. Stål, C.E.M. Wagner and G. Weiglein, MSSM Higgs Boson Searches at the LHC: Benchmark Scenarios after the Discovery of a Higgs-like Particle, Eur. Phys. J. C 73 (2013) 2552 [arXiv: 1302.7033] [INSPIRE].

[18] K. Lane and W. Shepherd, Natural stabilization of the Higgs boson's mass and alignment, Phys. Rev. D 99 (2019) 055015 [arXiv: 1808.07927] [INSPIRE].

[19] R. Harlander, M. Krämer and M. Schumacher, Bottom-quark associated Higgs-boson production: reconciling the four- and five-flavour scheme approach, CERN-PH-TH-2011-134 (2011) [arXiv: 1112.3478] [INSPIRE].

[20] LHC Higgs Cross Section Working Group, Handbook of LHC Higgs Cross Sections: 4. Deciphering the Nature of the Higgs Sector, arXiv:1610.07922 [INSPIRE].

[21] ALEPH collaboration, Search for charged Higgs bosons in $e^{+} e^{-}$collisions at energies up to $\sqrt{s}=209$ GeV, Phys. Lett. B 543 (2002) 1 [hep-ex/0207054] [INSPIRE].

[22] DELPHI collaboration, Search for charged Higgs bosons at LEP in general two Higgs doublet models, Eur. Phys. J. C 34 (2004) 399 [hep-ex/0404012] [INSPIRE].

[23] DELPHI collaboration, Search for charged Higgs bosons at LEP-2, Phys. Lett. B 460 (1999) 484 [INSPIRE].

[24] L3 collaboration, Search for charged Higgs bosons at LEP, Phys. Lett. B 575 (2003) 208 [hep-ex/0309056] [INSPIRE].

[25] OPAL collaboration, Search for Charged Higgs Bosons in $e^{+} e^{-}$Collisions at $\sqrt{s}=189-209 \mathrm{GeV}$, Eur. Phys. J. C 72 (2012) 2076 [arXiv:0812.0267] [INSPIRE].

[26] OPAL collaboration, Search for Higgs bosons in $e^{+} e^{-}$collisions at $183 \mathrm{GeV}$, Eur. Phys. J. C 7 (1999) 407 [hep-ex/9811025] [INSPIRE]. 
[27] ALEPH, DELPHI, L3, OPAL and LEP collaborations, Search for Charged Higgs bosons: Combined Results Using LEP Data, Eur. Phys. J. C 73 (2013) 2463 [arXiv:1301.6065] [INSPIRE].

[28] CDF collaboration, Search for Higgs bosons predicted in two-Higgs-doublet models via decays to tau lepton pairs in $1.96 \mathrm{TeV}$ pp collisions, Phys. Rev. Lett. 103 (2009) 201801 [arXiv: 0906.1014] [INSPIRE].

[29] D0 collaboration, Search for Higgs bosons of the minimal supersymmetric standard model in $p \bar{p}$ collisions at $\sqrt{s}=1.96 \mathrm{TeV}$, Phys. Lett. B 710 (2012) 569 [arXiv:1112.5431] [INSPIRE].

[30] A. Arbey, F. Mahmoudi, O. Stål and T. Stefaniak, Status of the Charged Higgs Boson in Two Higgs Doublet Models, Eur. Phys. J. C 78 (2018) 182 [arXiv:1706.07414] [InSPIRE].

[31] D0 collaboration, Search for charged Higgs bosons decaying to top and bottom quarks in p $\bar{p}$ collisions, Phys. Rev. Lett. 102 (2009) 191802 [arXiv:0807.0859] [INSPIRE].

[32] CMS collaboration, Search for a charged Higgs boson in pp collisions at $\sqrt{s}=8 \mathrm{TeV}$, JHEP 11 (2015) 018 [arXiv: 1508.07774] [INSPIRE].

[33] ATLAS collaboration, Search for charged Higgs bosons in the $H^{ \pm} \rightarrow$ tb decay channel in $p p$ collisions at $\sqrt{s}=8 \mathrm{TeV}$ using the ATLAS detector, JHEP 03 (2016) 127 [arXiv: 1512.03704] [INSPIRE].

[34] ATLAS collaboration, Search for charged Higgs bosons decaying into top and bottom quarks at $\sqrt{s}=13 \mathrm{TeV}$ with the ATLAS detector, JHEP 11 (2018) 085 [arXiv:1808.03599] [INSPIRE].

[35] ATLAS collaboration, Search for charged Higgs bosons decaying via $H^{ \pm} \rightarrow \tau^{ \pm} \nu_{\tau}$ in the $\tau+$ jets and $\tau+$ lepton final states with $36 \mathrm{fb}^{-1}$ of pp collision data recorded at $\sqrt{\mathrm{s}}=13 \mathrm{TeV}$ with the ATLAS experiment, JHEP 09 (2018) 139 [arXiv:1807.07915] [INSPIRE].

[36] ATLAS collaboration, Search for charged Higgs bosons produced in association with a top quark and decaying via $H^{ \pm} \rightarrow \tau \nu$ using pp collision data recorded at $\sqrt{s}=13 \mathrm{TeV}$ by the ATLAS detector, Phys. Lett. B 759 (2016) 555 [arXiv:1603.09203] [inSPIRE].

[37] ATLAS collaboration, Search for charged Higgs bosons decaying via $H^{ \pm} \rightarrow \tau^{ \pm} \nu$ in fully hadronic final states using pp collision data at $\sqrt{s}=8 \mathrm{TeV}$ with the ATLAS detector, JHEP 03 (2015) 088 [arXiv: 1412.6663] [INSPIRE].

[38] CMS collaboration, Search for a light charged Higgs boson decaying to c $\bar{s}$ in pp collisions at $\sqrt{s}=8 \mathrm{TeV}, J H E P 12(2015) 178$ [arXiv:1510.04252] [INSPIRE].

[39] CMS collaboration, Search for a charged Higgs boson decaying to charm and bottom quarks in proton-proton collisions at $\sqrt{s}=8 \mathrm{TeV}$, JHEP 11 (2018) 115 [arXiv:1808.06575] [INSPIRE].

[40] CMS collaboration, Search for Charged Higgs Bosons Produced via Vector Boson Fusion and Decaying into a Pair of $W$ and $Z$ Bosons Using pp Collisions at $\sqrt{s}=13 \mathrm{TeV}$, Phys. Rev. Lett. 119 (2017) 141802 [arXiv:1705.02942] [INSPIRE].

[41] CMS collaboration, Search for beyond the standard model Higgs bosons decaying into a $b \bar{b}$ pair in pp collisions at $\sqrt{s}=13 \mathrm{TeV}$, JHEP 08 (2018) 113 [arXiv:1805.12191] [INSPIRE].

[42] ATLAS collaboration, Search for a Charged Higgs Boson Produced in the Vector-Boson Fusion Mode with Decay $H^{ \pm} \rightarrow W^{ \pm} Z$ using pp Collisions at $\sqrt{s}=8$ TeV with the ATLAS Experiment, Phys. Rev. Lett. 114 (2015) 231801 [arXiv:1503.04233] [INSPIRE]. 
[43] CMS collaboration, Search for additional neutral MSSM Higgs bosons in the $\tau \tau$ final state in proton-proton collisions at $\sqrt{s}=13 \mathrm{TeV}, \mathrm{JHEP} 09$ (2018) 007 [arXiv:1803.06553] [INSPIRE].

[44] ATLAS collaboration, Search for additional heavy neutral Higgs and gauge bosons in the ditau final state produced in $36 \mathrm{fb}^{-1}$ of pp collisions at $\sqrt{s}=13 \mathrm{TeV}$ with the ATLAS detector, JHEP 01 (2018) 055 [arXiv:1709.07242] [INSPIRE].

[45] ATLAS collaboration, Search for Heavy Higgs Bosons A/H Decaying to a Top Quark Pair in pp Collisions at $\sqrt{s}=8 \mathrm{TeV}$ with the ATLAS Detector, Phys. Rev. Lett. 119 (2017) 191803 [arXiv: 1707.06025] [INSPIRE].

[46] CMS collaboration, Search for physics beyond the standard model in events with two leptons of same sign, missing transverse momentum and jets in proton-proton collisions at $\sqrt{s}=13$ TeV, Eur. Phys. J. C 77 (2017) 578 [arXiv:1704.07323] [InSPIRE].

[47] CMS collaboration, The CMS trigger system, 2017 JINST 12 P01020 [arXiv:1609.02366] [INSPIRE].

[48] CMS collaboration, The CMS Experiment at the CERN LHC, 2008 JINST 3 S08004 [INSPIRE].

[49] J. Alwall et al., The automated computation of tree-level and next-to-leading order differential cross sections and their matching to parton shower simulations, JHEP $\mathbf{0 7}$ (2014) 079 [arXiv: 1405.0301] [INSPIRE].

[50] S. Heinemeyer, W. Hollik and G. Weiglein, FeynHiggs: A Program for the calculation of the masses of the neutral CP even Higgs bosons in the MSSM, Comput. Phys. Commun. 124 (2000) 76 [hep-ph/9812320] [INSPIRE].

[51] E.L. Berger, T. Han, J. Jiang and T. Plehn, Associated production of a top quark and a charged Higgs boson, Phys. Rev. D 71 (2005) 115012 [hep-ph/0312286] [INSPIRE].

[52] M. Flechl, R. Klees, M. Krämer, M. Spira and M. Ubiali, Improved cross-section predictions for heavy charged Higgs boson production at the LHC, Phys. Rev. D 91 (2015) 075015 [arXiv:1409.5615] [INSPIRE].

[53] C. Degrande, M. Ubiali, M. Wiesemann and M. Zaro, Heavy charged Higgs boson production at the LHC, JHEP 10 (2015) 145 [arXiv: 1507.02549] [INSPIRE].

[54] S. Dittmaier, M. Krämer, M. Spira and M. Walser, Charged-Higgs-boson production at the LHC: NLO supersymmetric QCD corrections, Phys. Rev. D 83 (2011) 055005 [arXiv:0906.2648] [INSPIRE].

[55] A. Djouadi, J. Kalinowski and M. Spira, HDECAY: A Program for Higgs boson decays in the standard model and its supersymmetric extension, Comput. Phys. Commun. 108 (1998) 56 [hep-ph/9704448] [INSPIRE].

[56] S. Frixione, P. Nason and C. Oleari, Matching NLO QCD computations with Parton Shower simulations: the POWHEG method, JHEP 11 (2007) 070 [arXiv:0709.2092] [INSPIRE].

[57] P. Nason, A New method for combining NLO QCD with shower Monte Carlo algorithms, JHEP 11 (2004) 040 [hep-ph/0409146] [INSPIRE].

[58] S. Alioli, P. Nason, C. Oleari and E. Re, A general framework for implementing NLO calculations in shower Monte Carlo programs: the POWHEG BOX, JHEP 06 (2010) 043 [arXiv: 1002 .2581] [INSPIRE]. 
[59] J. Alwall et al., Comparative study of various algorithms for the merging of parton showers and matrix elements in hadronic collisions, Eur. Phys. J. C 53 (2008) 473 [arXiv:0706.2569] [INSPIRE].

[60] R. Frederix and S. Frixione, Merging meets matching in MC@NLO, JHEP 12 (2012) 061 [arXiv:1209.6215] [INSPIRE].

[61] NNPDF collaboration, Parton distributions for the LHC Run II, JHEP 04 (2015) 040 [arXiv:1410.8849] [INSPIRE].

[62] T. Sjöstrand et al., An Introduction to PYTHIA 8.2, Comput. Phys. Commun. 191 (2015) 159 [arXiv: 1410.3012] [INSPIRE].

[63] P. Skands, S. Carrazza and J. Rojo, Tuning PYTHIA 8.1: the Monash 2013 Tune, Eur. Phys. J. C 74 (2014) 3024 [arXiv: 1404.5630] [INSPIRE].

[64] CMS collaboration, Event generator tunes obtained from underlying event and multiparton scattering measurements, Eur. Phys. J. C 76 (2016) 155 [arXiv:1512.00815] [INSPIRE].

[65] M. Cacciari, M. Czakon, M. Mangano, A. Mitov and P. Nason, Top-pair production at hadron colliders with next-to-next-to-leading logarithmic soft-gluon resummation, Phys. Lett. B 710 (2012) 612 [arXiv:1111.5869] [INSPIRE].

[66] P. Bärnreuther, M. Czakon and A. Mitov, Percent Level Precision Physics at the Tevatron: First Genuine NNLO QCD Corrections to $q \bar{q} \rightarrow t \bar{t}+X$, Phys. Rev. Lett. 109 (2012) 132001 [arXiv: 1204.5201] [INSPIRE].

[67] M. Czakon and A. Mitov, NNLO corrections to top-pair production at hadron colliders: the all-fermionic scattering channels, JHEP 12 (2012) 054 [arXiv:1207.0236] [INSPIRE].

[68] M. Czakon and A. Mitov, NNLO corrections to top pair production at hadron colliders: the quark-gluon reaction, JHEP 01 (2013) 080 [arXiv: 1210.6832] [INSPIRE].

[69] M. Beneke, P. Falgari, S. Klein and C. Schwinn, Hadronic top-quark pair production with NNLL threshold resummation, Nucl. Phys. B 855 (2012) 695 [arXiv:1109.1536] [inSPIRE].

[70] M. Czakon, P. Fiedler and A. Mitov, Total Top-Quark Pair-Production Cross Section at Hadron Colliders Through $O\left(\alpha_{S}^{4}\right)$, Phys. Rev. Lett. 110 (2013) 252004 [arXiv:1303.6254] [INSPIRE].

[71] M. Czakon and A. Mitov, Top++: A Program for the Calculation of the Top-Pair Cross-Section at Hadron Colliders, Comput. Phys. Commun. 185 (2014) 2930 [arXiv:1112.5675] [INSPIRE].

[72] M. Aliev, H. Lacker, U. Langenfeld, S. Moch, P. Uwer and M. Wiedermann, HATHOR: HAdronic Top and Heavy quarks crOss section calculatoR, Comput. Phys. Commun. 182 (2011) 1034 [arXiv: 1007.1327] [INSPIRE].

[73] P. Kant et al., HatHor for single top-quark production: Updated predictions and uncertainty estimates for single top-quark production in hadronic collisions, Comput. Phys. Commun. 191 (2015) 74 [arXiv:1406.4403] [INSPIRE].

[74] F. Maltoni, D. Pagani and I. Tsinikos, Associated production of a top-quark pair with vector bosons at NLO in QCD: impact on $t \bar{t} H$ searches at the LHC, JHEP 02 (2016) 113 [arXiv: 1507. 05640] [INSPIRE].

[75] J.M. Campbell, R.K. Ellis and C. Williams, Vector boson pair production at the LHC, JHEP 07 (2011) 018 [arXiv:1105.0020] [INSPIRE]. 
[76] N. Kidonakis, Two-loop soft anomalous dimensions for single top quark associated production with a $W^{-}$or $H^{-}$, Phys. Rev. D 82 (2010) 054018 [arXiv:1005.4451] [INSPIRE].

[77] N. Bartosik, Associated Top-Quark-Pair and b-Jet Production in the Dilepton Channel at $\sqrt{s}=8 \mathrm{TeV}$ as Test of $Q C D$ and Background to $t+$ Higgs Production, Ph.D. Thesis, University of Hamburg, Hamburg Germany (2015) [DESY-THESIS-2015-035] [INSPIRE].

[78] M. Cacciari and G.P. Salam, Pileup subtraction using jet areas, Phys. Lett. B 659 (2008) 119 [arXiv:0707.1378] [INSPIRE].

[79] GEANT4 collaboration, GEANT4: A Simulation toolkit, Nucl. Instrum. Meth. A 506 (2003) 250 [INSPIRE].

[80] CMS collaboration, Particle-flow reconstruction and global event description with the CMS detector, 2017 JINST 12 P10003 [arXiv:1706.04965] [INSPIRE].

[81] CMS collaboration, Description and performance of track and primary-vertex reconstruction with the CMS tracker, 2014 JINST 9 P10009 [arXiv:1405.6569] [INSPIRE].

[82] M. Cacciari, G.P. Salam and G. Soyez, The anti- $k_{t}$ jet clustering algorithm, JHEP 04 (2008) 063 [arXiv:0802.1189] [InSPIRE].

[83] M. Cacciari, G.P. Salam and G. Soyez, FastJet User Manual, Eur. Phys. J. C 72 (2012) 1896 [arXiv: 1111.6097] [INSPIRE].

[84] CMS collaboration, Performance of Electron Reconstruction and Selection with the CMS Detector in Proton-Proton Collisions at $\sqrt{s}=8$ TeV, 2015 JINST 10 P06005 [arXiv: 1502.02701] [INSPIRE].

[85] CMS collaboration, Search for vector-like $T$ and $B$ quark pairs in final states with leptons at $\sqrt{s}=13$ TeV, JHEP 08 (2018) 177 [arXiv:1805.04758] [INSPIRE].

[86] CMS collaboration, Performance of the CMS muon detector and muon reconstruction with proton-proton collisions at $\sqrt{s}=13$ TeV, 2018 JINST 13 P06015 [arXiv:1804.04528] [INSPIRE].

[87] K. Rehermann and B. Tweedie, Efficient Identification of Boosted Semileptonic Top Quarks at the LHC, JHEP 03 (2011) 059 [arXiv: 1007.2221] [INSPIRE].

[88] CMS collaboration, Search for new physics in same-sign dilepton events in proton-proton collisions at $\sqrt{s}=13$ TeV, Eur. Phys. J. C 76 (2016) 439 [arXiv:1605.03171] [InSPIRE].

[89] CMS collaboration, Jet algorithms performance in 13 TeV data, CMS-PAS-JME-16-003 (2017) [INSPIRE].

[90] CMS collaboration, Jet energy scale and resolution in the CMS experiment in pp collisions at $8 \mathrm{TeV}, 2017$ JINST 12 P02014 [arXiv:1607.03663] [INSPIRE].

[91] CMS collaboration, Identification of heavy-flavour jets with the CMS detector in pp collisions at $13 \mathrm{TeV}, 2018$ JINST 13 P05011 [arXiv: 1712.07158] [INSPIRE].

[92] CMS collaboration, Performance of the CMS missing transverse momentum reconstruction in pp data at $\sqrt{s}=8 \mathrm{TeV}, 2015$ JINST $10 \mathrm{P} 02006$ [arXiv:1411.0511] [INSPIRE].

[93] CMS collaboration, Performance of reconstruction and identification of $\tau$ leptons decaying to hadrons and $\nu_{\tau}$ in pp collisions at $\sqrt{s}=13$ TeV, 2018 JINST 13 P10005 [arXiv: 1809.02816] [INSPIRE]. 
[94] CMS collaboration, Study of the Dijet Mass Spectrum in $p p \rightarrow W+$ Jets Events at $\sqrt{s}=7$ TeV, Phys. Rev. Lett. 109 (2012) 251801 [arXiv:1208.3477] [INSPIRE].

[95] CMS collaboration, Performance of CMS Muon Reconstruction in pp Collision Events at $\sqrt{s}=7 \mathrm{TeV}, 2012$ JINST 7 P10002 [arXiv:1206.4071] [INSPIRE].

[96] G.C. Fox and S. Wolfram, Observables for the Analysis of Event Shapes in $e^{+} e^{-}$ Annihilation and Other Processes, Phys. Rev. Lett. 41 (1978) 1581 [INSPIRE].

[97] T. Hastie, R. Tibshirani and J. Friedman, The elements of statistical learning, second edition, Springer Series in Statistics Series, Springer-Verlag (2013).

[98] L. Breiman, J. Friedman, R. Olshen and C. Stone, Classification and regression trees, Wadsworth (1984).

[99] H. Voss, A. Höcker, J. Stelzer and F. Tegenfeldt, TMVA, the toolkit for multivariate data analysis with ROOT, in proceedings of the 11th International Workshop on Advanced Computing and Analysis Techniques in Physics Research (ACAT 2007), Amsterdam, The Netherlands, 23-27 April 2007, p. 40 [PoS (ACAT) 040 (2007)] [INSPIRE].

[100] P. Baldi, K. Cranmer, T. Faucett, P. Sadowski and D. Whiteson, Parameterized neural networks for high-energy physics, Eur. Phys. J. C 76 (2016) 235 [arXiv:1601.07913] [INSPIRE].

[101] M. Abadi et al., TensorFlow: Large-Scale Machine Learning on Heterogeneous Distributed Systems, arXiv: 1603.04467 [INSPIRE].

[102] F. Chollet et al., Keras, (2015) https://keras.io/.

[103] ATLAS and CMS collaborations and the LHC Higgs Combination Group, Procedure for the LHC Higgs boson search combination in Summer 2011, CMS-NOTE-2011-005 (2011) [ATL-PHYS-PUB-2011-11] [INSPIRE].

[104] CMS collaboration, CMS Luminosity Measurements for the 2016 Data Taking Period, CMS-PAS-LUM-17-001 (2017) [INSPIRE].

[105] J. Butterworth et al., PDF 4LHC recommendations for LHC Run II, J. Phys. G 43 (2016) 023001 [arXiv: 1510.03865] [INSPIRE].

[106] CMS collaboration, Investigations of the impact of the parton shower tuning in PYTHIA 8 in the modelling of $t \bar{t}$ at $\sqrt{s}=8$ and $13 \mathrm{TeV}$, CMS-PAS-TOP-16-021 (2016) [INSPIRE].

[107] T. Ježo, J.M. Lindert, N. Moretti and S. Pozzorini, New NLOPS predictions for $t \bar{t}+b$-jet production at the LHC, Eur. Phys. J. C 78 (2018) 502 [arXiv: 1802.00426] [INSPIRE].

[108] R.J. Barlow and C. Beeston, Fitting using finite Monte Carlo samples, Comput. Phys. Commun. 77 (1993) 219 [INSPIRE].

[109] J.S. Conway, Incorporating Nuisance Parameters in Likelihoods for Multisource Spectra, in proceedings of the PHYSTAT 2011 Workshop on Statistical Issues Related to Discovery Claims in Search Experiments and Unfolding, CERN, Geneva, Switzerland, 17-20 January 2011, pp. 115-120 [arXiv:1103.0354] [INSPIRE].

[110] G. Cowan, K. Cranmer, E. Gross and O. Vitells, Asymptotic formulae for likelihood-based tests of new physics, Eur. Phys. J. C 71 (2011) 1554 [Erratum ibid. C 73 (2013) 2501] [arXiv: 1007.1727] [INSPIRE]. 
[111] A.L. Read, Presentation of search results: The $C L_{s}$ technique, J. Phys. G 28 (2002) 2693 [INSPIRE].

[112] T. Junk, Confidence level computation for combining searches with small statistics, Nucl. Instrum. Meth. A 434 (1999) 435 [hep-ex/9902006] [INSPIRE].

[113] H. Bahl et al., MSSM Higgs Boson Searches at the LHC: Benchmark Scenarios for Run 2 and Beyond, Eur. Phys. J. C 79 (2019) 617 [arXiv:1808.07542] [INSPIRE]. 


\section{The CMS collaboration}

Yerevan Physics Institute, Yerevan, Armenia

A.M. Sirunyan ${ }^{\dagger}$, A. Tumasyan

\section{Institut für Hochenergiephysik, Wien, Austria}

W. Adam, F. Ambrogi, T. Bergauer, J. Brandstetter, M. Dragicevic, J. Erö, A. Escalante Del Valle, M. Flechl, R. Frühwirth ${ }^{1}$, M. Jeitler ${ }^{1}$, N. Krammer, I. Krätschmer, D. Liko, T. Madlener, I. Mikulec, N. Rad, J. Schieck ${ }^{1}$, R. Schöfbeck, M. Spanring, D. Spitzbart, W. Waltenberger, C.-E. Wulz ${ }^{1}$, M. Zarucki

Institute for Nuclear Problems, Minsk, Belarus

V. Drugakov, V. Mossolov, J. Suarez Gonzalez

\section{Universiteit Antwerpen, Antwerpen, Belgium}

M.R. Darwish, E.A. De Wolf, D. Di Croce, X. Janssen, J. Lauwers, A. Lelek, M. Pieters, H. Rejeb Sfar, H. Van Haevermaet, P. Van Mechelen, S. Van Putte, N. Van Remortel

\section{Vrije Universiteit Brussel, Brussel, Belgium}

F. Blekman, E.S. Bols, S.S. Chhibra, J. D'Hondt, J. De Clercq, D. Lontkovskyi, S. Lowette, I. Marchesini, S. Moortgat, L. Moreels, Q. Python, K. Skovpen, S. Tavernier, W. Van Doninck, P. Van Mulders, I. Van Parijs

\section{Université Libre de Bruxelles, Bruxelles, Belgium}

D. Beghin, B. Bilin, H. Brun, B. Clerbaux, G. De Lentdecker, H. Delannoy, B. Dorney,

L. Favart, A. Grebenyuk, A.K. Kalsi, J. Luetic, A. Popov, N. Postiau, E. Starling,

L. Thomas, C. Vander Velde, P. Vanlaer, D. Vannerom, Q. Wang

\section{Ghent University, Ghent, Belgium}

T. Cornelis, D. Dobur, I. Khvastunov², C. Roskas, D. Trocino, M. Tytgat, W. Verbeke,

B. Vermassen, M. Vit, N. Zaganidis

Université Catholique de Louvain, Louvain-la-Neuve, Belgium

O. Bondu, G. Bruno, C. Caputo, P. David, C. Delaere, M. Delcourt, A. Giammanco, V. Lemaitre, A. Magitteri, J. Prisciandaro, A. Saggio, M. Vidal Marono, P. Vischia, J. Zobec

Centro Brasileiro de Pesquisas Fisicas, Rio de Janeiro, Brazil

F.L. Alves, G.A. Alves, G. Correia Silva, C. Hensel, A. Moraes, P. Rebello Teles

Universidade do Estado do Rio de Janeiro, Rio de Janeiro, Brazil

E. Belchior Batista Das Chagas, W. Carvalho, J. Chinellato ${ }^{3}$, E. Coelho, E.M. Da Costa, G.G. Da Silveira ${ }^{4}$, D. De Jesus Damiao, C. De Oliveira Martins, S. Fonseca De Souza, L.M. Huertas Guativa, H. Malbouisson, J. Martins ${ }^{5}$, D. Matos Figueiredo, M. Medina Jaime ${ }^{6}$, M. Melo De Almeida, C. Mora Herrera, L. Mundim, H. Nogima, W.L. Prado Da Silva, L.J. Sanchez Rosas, A. Santoro, A. Sznajder, M. Thiel, E.J. Tonelli Manganote ${ }^{3}$, F. Torres Da Silva De Araujo, A. Vilela Pereira 
Universidade Estadual Paulista ${ }^{a}$, Universidade Federal do $\mathrm{ABC}^{b}$, São Paulo, Brazil

S. Ahuja ${ }^{a}$, C.A. Bernardes ${ }^{a}$, L. Calligaris ${ }^{a}$, T.R. Fernandez Perez Tomei ${ }^{a}$, E.M. Gregores ${ }^{b}$, D.S. Lemos, P.G. Mercadante ${ }^{b}$, S.F. Novaes ${ }^{a}$, SandraS. Padula ${ }^{a}$

Institute for Nuclear Research and Nuclear Energy, Bulgarian Academy of Sciences, Sofia, Bulgaria

A. Aleksandrov, G. Antchev, R. Hadjiiska, P. Iaydjiev, A. Marinov, M. Misheva, M. Rodozov, M. Shopova, G. Sultanov

University of Sofia, Sofia, Bulgaria

M. Bonchev, A. Dimitrov, T. Ivanov, L. Litov, B. Pavlov, P. Petkov

Beihang University, Beijing, China

W. Fang $^{7}$, X. $\mathrm{Gao}^{7}$, L. Yuan

Institute of High Energy Physics, Beijing, China

M. Ahmad, G.M. Chen, H.S. Chen, M. Chen, C.H. Jiang, D. Leggat, H. Liao, Z. Liu, S.M. Shaheen ${ }^{8}$, A. Spiezia, J. Tao, E. Yazgan, H. Zhang, S. Zhang ${ }^{8}$, J. Zhao

State Key Laboratory of Nuclear Physics and Technology, Peking University, Beijing, China

A. Agapitos, Y. Ban, G. Chen, A. Levin, J. Li, L. Li, Q. Li, Y. Mao, S.J. Qian, D. Wang Tsinghua University, Beijing, China

Z. Hu, Y. Wang

Universidad de Los Andes, Bogota, Colombia

C. Avila, A. Cabrera, L.F. Chaparro Sierra, C. Florez, C.F. González Hernández, M.A. Segura Delgado

Universidad de Antioquia, Medellin, Colombia

J. Mejia Guisao, J.D. Ruiz Alvarez, C.A. Salazar González, N. Vanegas Arbelaez

University of Split, Faculty of Electrical Engineering, Mechanical Engineering and Naval Architecture, Split, Croatia

D. Giljanović, N. Godinovic, D. Lelas, I. Puljak, T. Sculac

University of Split, Faculty of Science, Split, Croatia

Z. Antunovic, M. Kovac

Institute Rudjer Boskovic, Zagreb, Croatia

V. Brigljevic, S. Ceci, D. Ferencek, K. Kadija, B. Mesic, M. Roguljic, A. Starodumov ${ }^{9}$, T. Susa

University of Cyprus, Nicosia, Cyprus

M.W. Ather, A. Attikis, E. Erodotou, A. Ioannou, M. Kolosova, S. Konstantinou, G. Mavromanolakis, J. Mousa, C. Nicolaou, F. Ptochos, P.A. Razis, H. Rykaczewski, D. Tsiakkouri 
Charles University, Prague, Czech Republic

M. Finger ${ }^{10}$, M. Finger Jr. ${ }^{10}$, A. Kveton, J. Tomsa

Escuela Politecnica Nacional, Quito, Ecuador

E. Ayala

Universidad San Francisco de Quito, Quito, Ecuador

E. Carrera Jarrin

Academy of Scientific Research and Technology of the Arab Republic of Egypt, Egyptian Network of High Energy Physics, Cairo, Egypt

H. Abdalla ${ }^{11}$, A.A. Abdelalim ${ }^{12,13}$

National Institute of Chemical Physics and Biophysics, Tallinn, Estonia

S. Bhowmik, A. Carvalho Antunes De Oliveira, R.K. Dewanjee, K. Ehataht, M. Kadastik, M. Raidal, C. Veelken

Department of Physics, University of Helsinki, Helsinki, Finland

P. Eerola, L. Forthomme, H. Kirschenmann, K. Osterberg, M. Voutilainen

Helsinki Institute of Physics, Helsinki, Finland

F. Garcia, J. Havukainen, J.K. Heikkilä, T. Järvinen, V. Karimäki, R. Kinnunen,

T. Lampén, K. Lassila-Perini, S. Laurila, S. Lehti, T. Lindén, P. Luukka, T. Mäenpää,

H. Siikonen, E. Tuominen, J. Tuominiemi

Lappeenranta University of Technology, Lappeenranta, Finland

T. Tuuva

IRFU, CEA, Université Paris-Saclay, Gif-sur-Yvette, France

M. Besancon, F. Couderc, M. Dejardin, D. Denegri, B. Fabbro, J.L. Faure, F. Ferri, S. Ganjour, A. Givernaud, P. Gras, G. Hamel de Monchenault, P. Jarry, C. Leloup, E. Locci, J. Malcles, J. Rander, A. Rosowsky, M.Ö. Sahin, A. Savoy-Navarro ${ }^{14}$, M. Titov

Laboratoire Leprince-Ringuet, CNRS/IN2P3, Ecole Polytechnique, Institut Polytechnique de Paris

C. Amendola, F. Beaudette, P. Busson, C. Charlot, B. Diab, G. Falmagne, R. Granier de Cassagnac, I. Kucher, A. Lobanov, C. Martin Perez, M. Nguyen, C. Ochando, P. Paganini, J. Rembser, R. Salerno, J.B. Sauvan, Y. Sirois, A. Zabi, A. Zghiche

Université de Strasbourg, CNRS, IPHC UMR 7178, Strasbourg, France

J.-L. Agram ${ }^{15}$, J. Andrea, D. Bloch, G. Bourgatte, J.-M. Brom, E.C. Chabert, C. Collard, E. Conte ${ }^{15}$, J.-C. Fontaine ${ }^{15}$, D. Gelé, U. Goerlach, M. Jansová, A.-C. Le Bihan, N. Tonon, P. Van Hove

Centre de Calcul de l'Institut National de Physique Nucleaire et de Physique des Particules, CNRS/IN2P3, Villeurbanne, France

S. Gadrat 
Université de Lyon, Université Claude Bernard Lyon 1, CNRS-IN2P3, Institut de Physique Nucléaire de Lyon, Villeurbanne, France

S. Beauceron, C. Bernet, G. Boudoul, C. Camen, N. Chanon, R. Chierici, D. Contardo, P. Depasse, H. El Mamouni, J. Fay, S. Gascon, M. Gouzevitch, B. Ille, Sa. Jain, F. Lagarde, I.B. Laktineh, H. Lattaud, M. Lethuillier, L. Mirabito, S. Perries, V. Sordini, G. Touquet, M. Vander Donckt, S. Viret

Georgian Technical University, Tbilisi, Georgia

A. Khvedelidze ${ }^{10}$

Tbilisi State University, Tbilisi, Georgia

Z. Tsamalaidze ${ }^{10}$

RWTH Aachen University, I. Physikalisches Institut, Aachen, Germany

C. Autermann, L. Feld, M.K. Kiesel, K. Klein, M. Lipinski, D. Meuser, A. Pauls, M. Preuten, M.P. Rauch, C. Schomakers, J. Schulz, M. Teroerde, B. Wittmer

RWTH Aachen University, III. Physikalisches Institut A, Aachen, Germany

A. Albert, M. Erdmann, S. Erdweg, T. Esch, B. Fischer, R. Fischer, S. Ghosh, T. Hebbeker, K. Hoepfner, H. Keller, L. Mastrolorenzo, M. Merschmeyer, A. Meyer, P. Millet, G. Mocellin, S. Mondal, S. Mukherjee, D. Noll, A. Novak, T. Pook, A. Pozdnyakov, T. Quast, M. Radziej, Y. Rath, H. Reithler, M. Rieger, J. Roemer, A. Schmidt, S.C. Schuler, A. Sharma, S. Thüer, S. Wiedenbeck

RWTH Aachen University, III. Physikalisches Institut B, Aachen, Germany G. Flügge, W. Haj Ahmad ${ }^{16}$, O. Hlushchenko, T. Kress, T. Müller, A. Nehrkorn, A. Nowack, C. Pistone, O. Pooth, D. Roy, H. Sert, A. Stahl ${ }^{17}$

Deutsches Elektronen-Synchrotron, Hamburg, Germany

M. Aldaya Martin, P. Asmuss, I. Babounikau, H. Bakhshiansohi, K. Beernaert, O. Behnke, U. Behrens, A. Bermúdez Martínez, D. Bertsche, A.A. Bin Anuar, K. Borras ${ }^{18}$, V. Botta, A. Campbell, A. Cardini, P. Connor, S. Consuegra Rodríguez, C. Contreras-Campana, V. Danilov, A. De Wit, M.M. Defranchis, C. Diez Pardos, D. Domínguez Damiani, G. Eckerlin, D. Eckstein, T. Eichhorn, A. Elwood, E. Eren, E. Gallo ${ }^{19}$, A. Geiser, J.M. Grados Luyando, A. Grohsjean, M. Guthoff, M. Haranko, A. Harb, A. Jafari, N.Z. Jomhari, H. Jung, A. Kasem ${ }^{18}$, M. Kasemann, H. Kaveh, J. Keaveney, C. Kleinwort, J. Knolle, D. Krücker, W. Lange, T. Lenz, J. Leonard, J. Lidrych, K. Lipka, W. Lohmann ${ }^{20}$, R. Mankel, I.-A. Melzer-Pellmann, A.B. Meyer, M. Meyer, M. Missiroli, G. Mittag, J. Mnich, A. Mussgiller, V. Myronenko, D. Pérez Adán, S.K. Pflitsch, D. Pitzl, A. Raspereza, A. Saibel, M. Savitskyi, V. Scheurer, P. Schütze, C. Schwanenberger, R. Shevchenko, A. Singh, H. Tholen, O. Turkot, A. Vagnerini, M. Van De Klundert, G.P. Van Onsem, R. Walsh, Y. Wen, K. Wichmann, C. Wissing, O. Zenaiev, R. Zlebcik

University of Hamburg, Hamburg, Germany

R. Aggleton, S. Bein, L. Benato, A. Benecke, V. Blobel, T. Dreyer, A. Ebrahimi, A. Fröhlich, C. Garbers, E. Garutti, D. Gonzalez, P. Gunnellini, J. Haller, A. Hinzmann, A. Karavdina, G. Kasieczka, R. Klanner, R. Kogler, N. Kovalchuk, S. Kurz, V. Kutzner, 
J. Lange, T. Lange, A. Malara, D. Marconi, J. Multhaup, M. Niedziela, C.E.N. Niemeyer, D. Nowatschin, A. Perieanu, A. Reimers, O. Rieger, C. Scharf, P. Schleper, S. Schumann, J. Schwandt, J. Sonneveld, H. Stadie, G. Steinbrück, F.M. Stober, M. Stöver, B. Vormwald, I. Zoi

Karlsruher Institut fuer Technologie, Karlsruhe, Germany

M. Akbiyik, C. Barth, M. Baselga, S. Baur, T. Berger, E. Butz, R. Caspart, T. Chwalek, W. De Boer, A. Dierlamm, K. El Morabit, N. Faltermann, M. Giffels, P. Goldenzweig, A. Gottmann, M.A. Harrendorf, F. Hartmann ${ }^{17}$, U. Husemann, S. Kudella, S. Mitra, M.U. Mozer, Th. Müller, M. Musich, A. Nürnberg, G. Quast, K. Rabbertz, M. Schröder, I. Shvetsov, H.J. Simonis, R. Ulrich, M. Weber, C. Wöhrmann, R. Wolf

Institute of Nuclear and Particle Physics (INPP), NCSR Demokritos, Aghia Paraskevi, Greece

G. Anagnostou, P. Asenov, G. Daskalakis, T. Geralis, A. Kyriakis, D. Loukas, G. Paspalaki National and Kapodistrian University of Athens, Athens, Greece

M. Diamantopoulou, G. Karathanasis, P. Kontaxakis, A. Panagiotou, I. Papavergou, N. Saoulidou, A. Stakia, K. Theofilatos, K. Vellidis

National Technical University of Athens, Athens, Greece

G. Bakas, K. Kousouris, I. Papakrivopoulos, G. Tsipolitis

University of Ioánnina, Ioánnina, Greece

I. Evangelou, C. Foudas, P. Gianneios, P. Katsoulis, P. Kokkas, S. Mallios, K. Manitara, N. Manthos, I. Papadopoulos, J. Strologas, F.A. Triantis, D. Tsitsonis

MTA-ELTE Lendület CMS Particle and Nuclear Physics Group, Eötvös Loránd University, Budapest, Hungary

M. Bartók ${ }^{21}$, M. Csanad, P. Major, K. Mandal, A. Mehta, M.I. Nagy, G. Pasztor, O. Surányi, G.I. Veres

Wigner Research Centre for Physics, Budapest, Hungary

G. Bencze, C. Hajdu, D. Horvath ${ }^{22}$, F. Sikler, T.Á. Vámi, V. Veszpremi, G. Vesztergombi ${ }^{\dagger}$

Institute of Nuclear Research ATOMKI, Debrecen, Hungary

N. Beni, S. Czellar, J. Karancsi ${ }^{21}$, A. Makovec, J. Molnar, Z. Szillasi

Institute of Physics, University of Debrecen, Debrecen, Hungary

P. Raics, D. Teyssier, Z.L. Trocsanyi, B. Ujvari

Eszterhazy Karoly University, Karoly Robert Campus, Gyongyos, Hungary

T. Csorgo, W.J. Metzger, F. Nemes, T. Novak

Indian Institute of Science (IISc), Bangalore, India

S. Choudhury, J.R. Komaragiri, P.C. Tiwari 
National Institute of Science Education and Research, HBNI, Bhubaneswar, India

S. Bahinipati ${ }^{24}$, C. Kar, G. Kole, P. Mal, V.K. Muraleedharan Nair Bindhu, A. Nayak ${ }^{25}$, D.K. Sahoo ${ }^{24}$, S.K. Swain

Panjab University, Chandigarh, India

S. Bansal, S.B. Beri, V. Bhatnagar, S. Chauhan, R. Chawla, N. Dhingra, R. Gupta, A. Kaur, M. Kaur, S. Kaur, P. Kumari, M. Lohan, M. Meena, K. Sandeep, S. Sharma, J.B. Singh, A.K. Virdi

University of Delhi, Delhi, India

A. Bhardwaj, B.C. Choudhary, R.B. Garg, M. Gola, S. Keshri, Ashok Kumar, S. Malhotra, M. Naimuddin, P. Priyanka, K. Ranjan, Aashaq Shah, R. Sharma

Saha Institute of Nuclear Physics, HBNI, Kolkata, India

R. Bhardwaj ${ }^{26}$, M. Bharti ${ }^{26}$, R. Bhattacharya, S. Bhattacharya, U. Bhawandeep ${ }^{26}$, D. Bhowmik, S. Dey, S. Dutta, S. Ghosh, M. Maity ${ }^{27}$, K. Mondal, S. Nandan, A. Purohit, P.K. Rout, G. Saha, S. Sarkar, T. Sarkar ${ }^{27}$, M. Sharan, B. Singh ${ }^{26}$, S. Thakur ${ }^{26}$

Indian Institute of Technology Madras, Madras, India

P.K. Behera, P. Kalbhor, A. Muhammad, P.R. Pujahari, A. Sharma, A.K. Sikdar

Bhabha Atomic Research Centre, Mumbai, India

R. Chudasama, D. Dutta, V. Jha, V. Kumar, D.K. Mishra, P.K. Netrakanti, L.M. Pant, P. Shukla

Tata Institute of Fundamental Research-A, Mumbai, India

T. Aziz, M.A. Bhat, S. Dugad, G.B. Mohanty, N. Sur, RavindraKumar Verma

Tata Institute of Fundamental Research-B, Mumbai, India

S. Banerjee, S. Bhattacharya, S. Chatterjee, P. Das, M. Guchait, S. Karmakar, S. Kumar, G. Majumder, K. Mazumdar, N. Sahoo, S. Sawant

Indian Institute of Science Education and Research (IISER), Pune, India

S. Chauhan, S. Dube, V. Hegde, A. Kapoor, K. Kothekar, S. Pandey, A. Rane, A. Rastogi, S. Sharma

Institute for Research in Fundamental Sciences (IPM), Tehran, Iran

S. Chenarani ${ }^{28}$, E. Eskandari Tadavani, S.M. Etesami ${ }^{28}$, M. Khakzad, M. Mohammadi Najafabadi, M. Naseri, F. Rezaei Hosseinabadi

University College Dublin, Dublin, Ireland

M. Felcini, M. Grunewald

INFN Sezione di Bari ${ }^{a}$, Università di Bari ${ }^{b}$, Politecnico di Bari ${ }^{c}$, Bari, Italy

M. Abbrescia ${ }^{a, b}$, C. Calabria ${ }^{a, b}$, A. Colaleo ${ }^{a}$, D. Creanza ${ }^{a, c}$, L. Cristella ${ }^{a, b}$, N. De Filippis ${ }^{a, c}$, M. De Palma ${ }^{a, b}$, A. Di Florio ${ }^{a, b}$, L. Fiore $^{a}$, A. Gelmi $^{a, b}$, G. Iaselli $^{a, c}$, M. Ince ${ }^{a, b}$, S. Lezki ${ }^{a, b}$, G. Maggi ${ }^{a, c}$, M. Maggi ${ }^{a}$, G. Miniello ${ }^{a, b}$, S. My ${ }^{a, b}$, S. Nuzzo $^{a, b}$, 


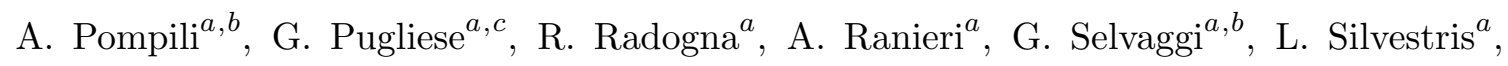
R. Venditti ${ }^{a}$, P. Verwilligen ${ }^{a}$

INFN Sezione di Bologna ${ }^{a}$, Università di Bologna ${ }^{b}$, Bologna, Italy

G. Abbiendi ${ }^{a}$, C. Battilana ${ }^{a, b}$, D. Bonacorsi ${ }^{a, b}$, L. Borgonovi $^{a, b}$, S. Braibant-Giacomelli $^{a, b}$, R. Campanini ${ }^{a, b}$, P. Capiluppi ${ }^{a, b}$, A. Castro ${ }^{a, b}$, F.R. Cavallo ${ }^{a},{\text { C. } \text { Ciocca }^{a}, \text { G. Codispoti }^{a, b}}^{,}$, M. Cuffiani ${ }^{a, b}$, G.M. Dallavalle ${ }^{a}$, F. Fabbri ${ }^{a}$, A. Fanfani ${ }^{a, b}$, E. Fontanesi, P. Giacomelli ${ }^{a}$, C. Grandi ${ }^{a}$, L. Guiducci ${ }^{a, b}$, F. Iemmi ${ }^{a, b}$, S. Lo Meo ${ }^{a, 29}$, S. Marcellini ${ }^{a}$, G. Masetti ${ }^{a}$,

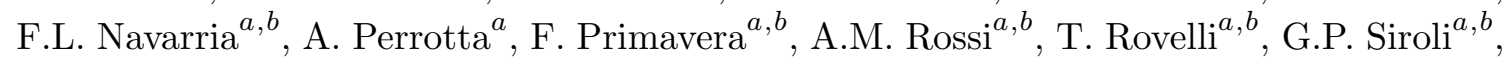
N. Tosi ${ }^{a}$

INFN Sezione di Catania ${ }^{a}$, Università di Catania ${ }^{b}$, Catania, Italy

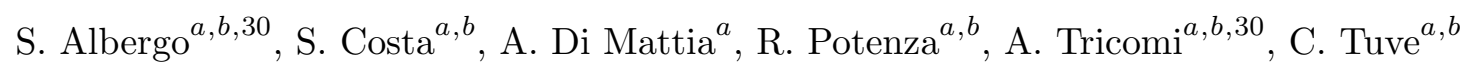

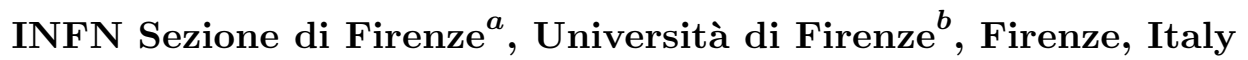

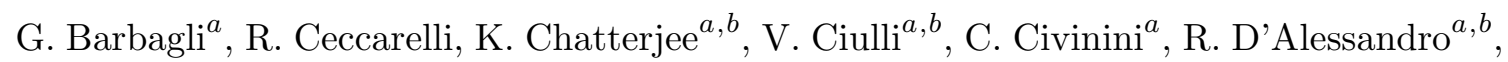

E. Focardi ${ }^{a, b}$, G. Latino, P. Lenzi ${ }^{a, b}$, M. Meschini $^{a}$, S. Paoletti $^{a}$, G. Sguazzoni $^{a}$, D. Strom ${ }^{a}$, L. Viliani ${ }^{a}$

INFN Laboratori Nazionali di Frascati, Frascati, Italy

L. Benussi, S. Bianco, D. Piccolo

INFN Sezione di Genova ${ }^{a}$, Università di Genova ${ }^{b}$, Genova, Italy

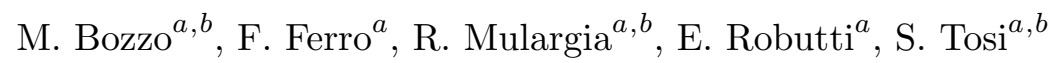

INFN Sezione di Milano-Bicocca ${ }^{a}$, Università di Milano-Bicocca ${ }^{b}$, Milano, Italy

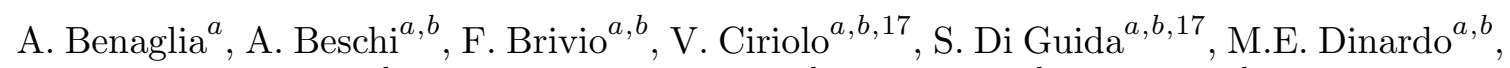

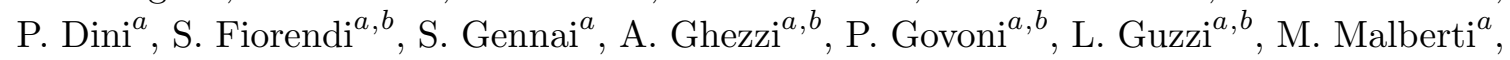

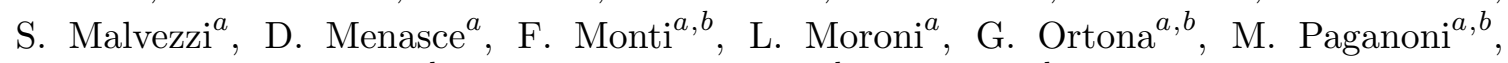

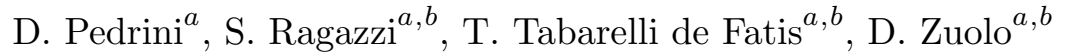

INFN Sezione di Napoli ${ }^{a}$, Università di Napoli 'Federico II'b , Napoli, Italy, Università della Basilicata $^{c}$, Potenza, Italy, Università G. Marconi ${ }^{d}$, Roma, Italy

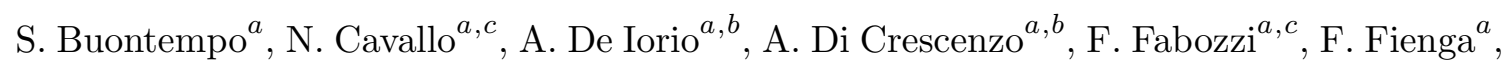
G. Galati ${ }^{a}$, A.O.M. Iorio ${ }^{a, b}$, L. Lista ${ }^{a, b}$, S. Meola ${ }^{a, d, 17}$, P. Paolucci ${ }^{a, 17}$, B. Rossi ${ }^{a}$, C. Sciacca ${ }^{a, b}$, E. Voevodina ${ }^{a, b}$

INFN Sezione di Padova ${ }^{a}$, Università di Padova ${ }^{b}$, Padova, Italy, Università di Trento $^{c}$, Trento, Italy

P. Azzi ${ }^{a}$, N. Bacchetta ${ }^{a}$, A. Boletti ${ }^{a, b}$, A. Bragagnolo, R. Carlin ${ }^{a, b}$, P. Checchia ${ }^{a}$, P. De Castro Manzano ${ }^{a}$, T. Dorigo ${ }^{a}$, U. Dosselli ${ }^{a}$, F. Gasparini ${ }^{a}, b$, U. Gasparini ${ }^{a, b}$, A. Gozzelino ${ }^{a}$, S.Y. Hoh, P. Lujan, M. Margoni ${ }^{a, b}$, A.T. Meneguzzo ${ }^{a, b}$, J. Pazzini ${ }^{a, b}$, N. Pozzobon ${ }^{a, b}$, M. Presilla ${ }^{b}$, P. Ronchese ${ }^{a, b}$, R. Rossin ${ }^{a, b}$, F. Simonetto ${ }^{a, b}$, A. Tiko, M. Tosi ${ }^{a, b}$, M. Zanetti ${ }^{a, b}$, P. Zotto ${ }^{a, b}$, G. Zumerle ${ }^{a, b}$ 
INFN Sezione di Pavia ${ }^{a}$, Università di Pavia ${ }^{b}$, Pavia, Italy

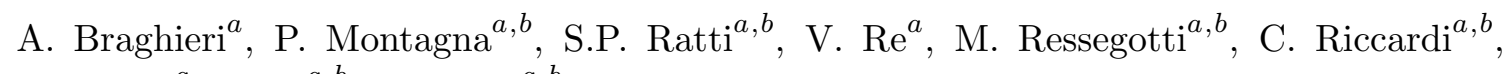
P. Salvini ${ }^{a}$, I. Vai ${ }^{a, b}$, P. Vitulo ${ }^{a, b}$

INFN Sezione di Perugia ${ }^{a}$, Università di Perugia ${ }^{b}$, Perugia, Italy

M. Biasini ${ }^{a, b}$, G.M. Bilei ${ }^{a}$, C. Cecchi ${ }^{a, b}$, D. Ciangottini ${ }^{a, b}$, L. Fanò $^{a, b}$, P. Lariccia $^{a, b}$,

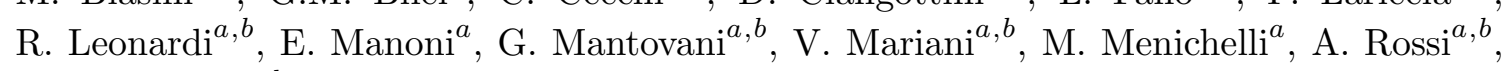
A. Santocchia ${ }^{a, b}$, D. Spiga ${ }^{a}$

INFN Sezione di Pisa ${ }^{a}$, Università di Pisa ${ }^{b}$, Scuola Normale Superiore di Pisa ${ }^{c}$, Pisa, Italy

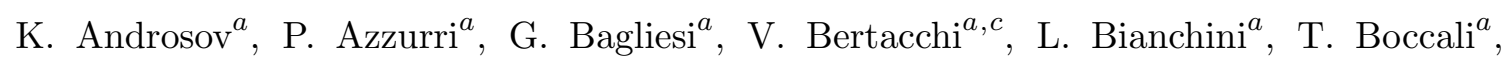

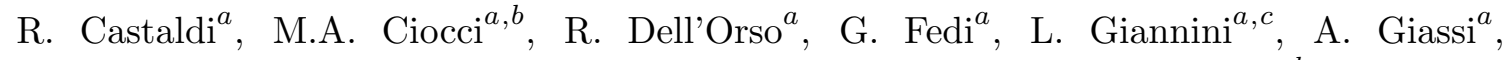
M.T. Grippo ${ }^{a}$, F. Ligabue ${ }^{a, c}$, E. Manca ${ }^{a, c}$, G. Mandorli ${ }^{a, c}$, A. Messineo ${ }^{a, b}$, F. Palla $^{a}$, A. Rizzi ${ }^{a}, b$ G. Rolandi ${ }^{31}$, S. Roy Chowdhury, A. Scribano ${ }^{a}$, P. Spagnolo $^{a}$, R. Tenchini ${ }^{a}$, G. Tonelli ${ }^{a, b}$, N. Turini, A. Venturi ${ }^{a}$, P.G. Verdini ${ }^{a}$

INFN Sezione di Roma ${ }^{a}$, Sapienza Università di Roma ${ }^{b}$, Rome, Italy

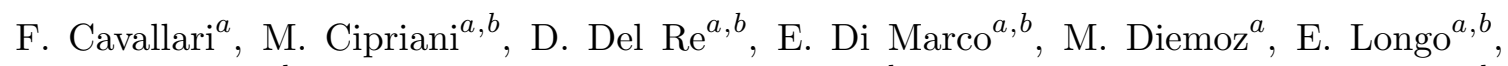
B. Marzocchi $^{a, b}$, P. Meridiani ${ }^{a}$, G. Organtini ${ }^{a, b}$, F. Pandolfi ${ }^{a}$, R. Paramatti ${ }^{a, b}$,

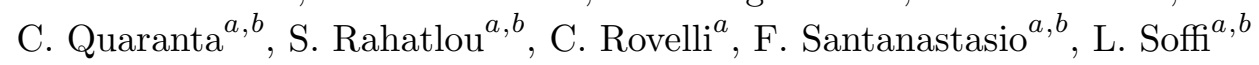

INFN Sezione di Torino ${ }^{a}$, Università di Torino ${ }^{b}$, Torino, Italy, Università del Piemonte Orientale ${ }^{c}$, Novara, Italy

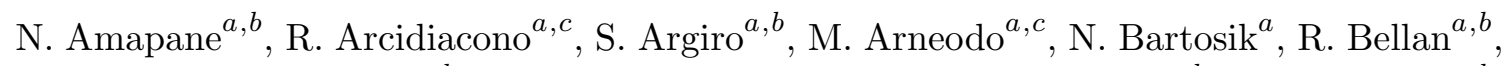

C. Biino ${ }^{a}$, A. Cappati ${ }^{a, b}$, N. Cartiglia ${ }^{a}$, S. Cometti ${ }^{a}$, M. Costa $^{a, b}$, R. Covarelli ${ }^{a, b}$,

N. Demaria ${ }^{a}$, B. Kiani ${ }^{a, b}$, C. Mariotti $^{a}$, S. Maselli ${ }^{a}$, E. Migliore ${ }^{a, b}$, V. Monaco ${ }^{a, b}$,

E. Monteil $^{a, b}$, M. Monteno ${ }^{a}$, M.M. Obertino ${ }^{a, b}$, L. Pacher $^{a, b}$, N. Pastrone $^{a}$, M. Pelliccioni ${ }^{a}$, G.L. Pinna Angioni ${ }^{a, b}$, A. Romero ${ }^{a, b}$, M. Ruspa $^{a, c}$, R. Sacchi $^{a, b}$, R. Salvatico $^{a, b}$, V. Sola $^{a}$, A. Solano ${ }^{a, b}$, D. Soldi ${ }^{a, b}$, A. Staiano ${ }^{a}$

INFN Sezione di Trieste ${ }^{a}$, Università di Trieste ${ }^{b}$, Trieste, Italy

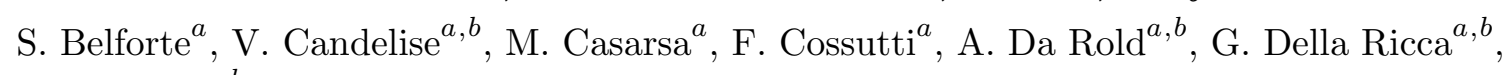
F. Vazzoler ${ }^{a, b}$, A. Zanetti ${ }^{a}$

Kyungpook National University, Daegu, Korea

B. Kim, D.H. Kim, G.N. Kim, M.S. Kim, J. Lee, S.W. Lee, C.S. Moon, Y.D. Oh, S.I. Pak, S. Sekmen, D.C. Son, Y.C. Yang

Chonnam National University, Institute for Universe and Elementary Particles, Kwangju, Korea

H. Kim, D.H. Moon, G. Oh

Hanyang University, Seoul, Korea

B. Francois, T.J. Kim, J. Park 
Korea University, Seoul, Korea

S. Cho, S. Choi, Y. Go, D. Gyun, S. Ha, B. Hong, K. Lee, K.S. Lee, J. Lim, J. Park, S.K. Park, Y. Roh

Kyung Hee University, Department of Physics

J. Goh

Sejong University, Seoul, Korea

H.S. Kim

Seoul National University, Seoul, Korea

J. Almond, J.H. Bhyun, J. Choi, S. Jeon, J. Kim, J.S. Kim, H. Lee, K. Lee, S. Lee, K. Nam,

M. Oh, S.B. Oh, B.C. Radburn-Smith, U.K. Yang, H.D. Yoo, I. Yoon, G.B. Yu

University of Seoul, Seoul, Korea

D. Jeon, H. Kim, J.H. Kim, J.S.H. Lee, I.C. Park, I. Watson

Sungkyunkwan University, Suwon, Korea

Y. Choi, C. Hwang, Y. Jeong, J. Lee, Y. Lee, I. Yu

Riga Technical University, Riga, Latvia

V. Veckalns ${ }^{32}$

Vilnius University, Vilnius, Lithuania

V. Dudenas, A. Juodagalvis, J. Vaitkus

National Centre for Particle Physics, Universiti Malaya, Kuala Lumpur, Malaysia

Z.A. Ibrahim, F. Mohamad Idris ${ }^{33}$, W.A.T. Wan Abdullah, M.N. Yusli, Z. Zolkapli

Universidad de Sonora (UNISON), Hermosillo, Mexico

J.F. Benitez, A. Castaneda Hernandez, J.A. Murillo Quijada, L. Valencia Palomo

Centro de Investigacion y de Estudios Avanzados del IPN, Mexico City, Mexico

H. Castilla-Valdez, E. De La Cruz-Burelo, I. Heredia-De La Cruz ${ }^{34}$, R. Lopez-Fernandez,

A. Sanchez-Hernandez

Universidad Iberoamericana, Mexico City, Mexico

S. Carrillo Moreno, C. Oropeza Barrera, M. Ramirez-Garcia, F. Vazquez Valencia

Benemerita Universidad Autonoma de Puebla, Puebla, Mexico

J. Eysermans, I. Pedraza, H.A. Salazar Ibarguen, C. Uribe Estrada

Universidad Autónoma de San Luis Potosí, San Luis Potosí, Mexico

A. Morelos Pineda

University of Montenegro, Podgorica, Montenegro

N. Raicevic

University of Auckland, Auckland, New Zealand

D. Krofcheck 
University of Canterbury, Christchurch, New Zealand

S. Bheesette, P.H. Butler

National Centre for Physics, Quaid-I-Azam University, Islamabad, Pakistan

A. Ahmad, M. Ahmad, Q. Hassan, H.R. Hoorani, W.A. Khan, M.A. Shah, M. Shoaib, M. Waqas

AGH University of Science and Technology Faculty of Computer Science, Electronics and Telecommunications, Krakow, Poland

V. Avati, L. Grzanka, M. Malawski

National Centre for Nuclear Research, Swierk, Poland

H. Bialkowska, M. Bluj, B. Boimska, M. Górski, M. Kazana, M. Szleper, P. Zalewski

Institute of Experimental Physics, Faculty of Physics, University of Warsaw, Warsaw, Poland

K. Bunkowski, A. Byszuk ${ }^{35}$, K. Doroba, A. Kalinowski, M. Konecki, J. Krolikowski, M. Misiura, M. Olszewski, A. Pyskir, M. Walczak

Laboratório de Instrumentação e Física Experimental de Partículas, Lisboa, Portugal

M. Araujo, P. Bargassa, D. Bastos, A. Di Francesco, P. Faccioli, B. Galinhas, M. Gallinaro, J. Hollar, N. Leonardo, J. Seixas, K. Shchelina, G. Strong, O. Toldaiev, J. Varela

Joint Institute for Nuclear Research, Dubna, Russia

V. Alexakhin, P. Bunin, Y. Ershov, I. Golutvin, I. Gorbunov, V. Karjavine, V. Korenkov, A. Lanev, A. Malakhov, V. Matveev ${ }^{36,37}$, P. Moisenz, V. Palichik, V. Perelygin, M. Savina, S. Shmatov, S. Shulha, O. Teryaev, N. Voytishin, B.S. Yuldashev ${ }^{38}$, A. Zarubin

Petersburg Nuclear Physics Institute, Gatchina (St. Petersburg), Russia

L. Chtchipounov, V. Golovtsov, Y. Ivanov, V. Kim ${ }^{39}$, E. Kuznetsova ${ }^{40}$, P. Levchenko, V. Murzin, V. Oreshkin, I. Smirnov, D. Sosnov, V. Sulimov, L. Uvarov, A. Vorobyev

Institute for Nuclear Research, Moscow, Russia

Yu. Andreev, A. Dermenev, S. Gninenko, N. Golubev, A. Karneyeu, M. Kirsanov, N. Krasnikov, A. Pashenkov, D. Tlisov, A. Toropin

Institute for Theoretical and Experimental Physics named by A.I. Alikhanov of NRC 'Kurchatov Institute', Moscow, Russia

V. Epshteyn, V. Gavrilov, N. Lychkovskaya, A. Nikitenko ${ }^{41}$, V. Popov, I. Pozdnyakov, G. Safronov, A. Spiridonov, A. Stepennov, M. Toms, E. Vlasov, A. Zhokin

Moscow Institute of Physics and Technology, Moscow, Russia

T. Aushev

National Research Nuclear University 'Moscow Engineering Physics Institute' (MEPhI), Moscow, Russia

O. Bychkova, R. Chistov ${ }^{42}$, M. Danilov ${ }^{42}$, S. Polikarpov ${ }^{42}$, E. Tarkovskii 
P.N. Lebedev Physical Institute, Moscow, Russia

V. Andreev, M. Azarkin, I. Dremin, M. Kirakosyan, A. Terkulov

Skobeltsyn Institute of Nuclear Physics, Lomonosov Moscow State University, Moscow, Russia

A. Baskakov, A. Belyaev, E. Boos, V. Bunichev, M. Dubinin ${ }^{43}$, L. Dudko, V. Klyukhin,

O. Kodolova, I. Lokhtin, S. Obraztsov, M. Perfilov, S. Petrushanko, V. Savrin

Novosibirsk State University (NSU), Novosibirsk, Russia

A. Barnyakov ${ }^{44}$, V. Blinov ${ }^{44}$, T. Dimova ${ }^{44}$, L. Kardapoltsev ${ }^{44}$, Y. Skovpen ${ }^{44}$

Institute for High Energy Physics of National Research Centre 'Kurchatov Institute', Protvino, Russia

I. Azhgirey, I. Bayshev, S. Bitioukov, V. Kachanov, D. Konstantinov, P. Mandrik,

V. Petrov, R. Ryutin, S. Slabospitskii, A. Sobol, S. Troshin, N. Tyurin, A. Uzunian,

A. Volkov

National Research Tomsk Polytechnic University, Tomsk, Russia

A. Babaev, A. Iuzhakov, V. Okhotnikov

Tomsk State University, Tomsk, Russia

V. Borchsh, V. Ivanchenko, E. Tcherniaev

University of Belgrade: Faculty of Physics and VINCA Institute of Nuclear Sciences

P. Adzic ${ }^{45}$, P. Cirkovic, D. Devetak, M. Dordevic, P. Milenovic, J. Milosevic, M. Stojanovic

Centro de Investigaciones Energéticas Medioambientales y Tecnológicas (CIEMAT), Madrid, Spain

M. Aguilar-Benitez, J. Alcaraz Maestre, A. Álvarez Fernández, I. Bachiller, M. Barrio Luna, J.A. Brochero Cifuentes, C.A. Carrillo Montoya, M. Cepeda, M. Cerrada, N. Colino, B. De La Cruz, A. Delgado Peris, C. Fernandez Bedoya, J.P. Fernández Ramos, J. Flix, M.C. Fouz, O. Gonzalez Lopez, S. Goy Lopez, J.M. Hernandez, M.I. Josa, D. Moran, Á. Navarro Tobar, A. Pérez-Calero Yzquierdo, J. Puerta Pelayo, I. Redondo, L. Romero, S. Sánchez Navas, M.S. Soares, A. Triossi, C. Willmott

Universidad Autónoma de Madrid, Madrid, Spain

C. Albajar, J.F. de Trocóniz

Universidad de Oviedo, Instituto Universitario de Ciencias y Tecnologías Espaciales de Asturias (ICTEA), Oviedo, Spain

B. Alvarez Gonzalez, J. Cuevas, C. Erice, J. Fernandez Menendez, S. Folgueras, I. Gonzalez Caballero, J.R. González Fernández, E. Palencia Cortezon, V. Rodríguez Bouza, S. Sanchez Cruz

Instituto de Física de Cantabria (IFCA), CSIC-Universidad de Cantabria, Santander, Spain

I.J. Cabrillo, A. Calderon, B. Chazin Quero, J. Duarte Campderros, M. Fernandez, P.J. Fernández Manteca, A. García Alonso, G. Gomez, C. Martinez Rivero, P. Mar- 
tinez Ruiz del Arbol, F. Matorras, J. Piedra Gomez, C. Prieels, T. Rodrigo, A. Ruiz-Jimeno, L. Russo $^{46}$, L. Scodellaro, N. Trevisani, I. Vila, J.M. Vizan Garcia

\section{University of Colombo, Colombo, Sri Lanka}

K. Malagalage

\section{University of Ruhuna, Department of Physics, Matara, Sri Lanka}

W.G.D. Dharmaratna, N. Wickramage

\section{CERN, European Organization for Nuclear Research, Geneva, Switzerland}

D. Abbaneo, B. Akgun, E. Auffray, G. Auzinger, J. Baechler, P. Baillon, A.H. Ball, D. Barney, J. Bendavid, M. Bianco, A. Bocci, E. Bossini, C. Botta, E. Brondolin, T. Camporesi, A. Caratelli, G. Cerminara, E. Chapon, G. Cucciati, D. d'Enterria, A. Dabrowski, N. Daci, V. Daponte, A. David, O. Davignon, A. De Roeck, N. Deelen, M. Deile, M. Dobson, M. Dünser, N. Dupont, A. Elliott-Peisert, F. Fallavollita ${ }^{47}$, D. Fasanella, G. Franzoni, J. Fulcher, W. Funk, S. Giani, D. Gigi, A. Gilbert, K. Gill, F. Glege, M. Gruchala, M. Guilbaud, D. Gulhan, J. Hegeman, C. Heidegger, Y. Iiyama, V. Innocente, P. Janot, O. Karacheban ${ }^{20}$, J. Kaspar, J. Kieseler, M. Krammer ${ }^{1}$, C. Lange, P. Lecoq, C. Lourenço, L. Malgeri, M. Mannelli, A. Massironi, F. Meijers, J.A. Merlin, S. Mersi, E. Meschi, F. Moortgat, M. Mulders, J. Ngadiuba, S. Nourbakhsh, S. Orfanelli, L. Orsini, F. Pantaleo ${ }^{17}$, L. Pape, E. Perez, M. Peruzzi, A. Petrilli, G. Petrucciani, A. Pfeiffer, M. Pierini, F.M. Pitters, D. Rabady, A. Racz, M. Rovere, H. Sakulin, C. Schäfer, C. Schwick, M. Selvaggi, A. Sharma, P. Silva, W. Snoeys, P. Sphicas ${ }^{48}$, J. Steggemann, V.R. Tavolaro, D. Treille, A. Tsirou, A. Vartak, M. Verzetti, W.D. Zeuner

\section{Paul Scherrer Institut, Villigen, Switzerland}

L. Caminada ${ }^{49}$, K. Deiters, W. Erdmann, R. Horisberger, Q. Ingram, H.C. Kaestli, D. Kotlinski, U. Langenegger, T. Rohe, S.A. Wiederkehr

ETH Zurich - Institute for Particle Physics and Astrophysics (IPA), Zurich, Switzerland

M. Backhaus, P. Berger, N. Chernyavskaya, G. Dissertori, M. Dittmar, M. Donegà, C. Dorfer, T.A. Gómez Espinosa, C. Grab, D. Hits, T. Klijnsma, W. Lustermann, R.A. Manzoni, M. Marionneau, M.T. Meinhard, F. Micheli, P. Musella, F. Nessi-Tedaldi, F. Pauss, G. Perrin, L. Perrozzi, S. Pigazzini, M. Reichmann, C. Reissel, T. Reitenspiess, D. Ruini, D.A. Sanz Becerra, M. Schönenberger, L. Shchutska, M.L. Vesterbacka Olsson, R. Wallny, D.H. Zhu

\section{Universität Zürich, Zurich, Switzerland}

T.K. Aarrestad, C. Amsler ${ }^{50}$, D. Brzhechko, M.F. Canelli, A. De Cosa, R. Del Burgo, S. Donato, B. Kilminster, S. Leontsinis, V.M. Mikuni, I. Neutelings, G. Rauco, P. Robmann, D. Salerno, K. Schweiger, C. Seitz, Y. Takahashi, S. Wertz, A. Zucchetta

\section{National Central University, Chung-Li, Taiwan}

T.H. Doan, C.M. Kuo, W. Lin, A. Roy, S.S. Yu 
National Taiwan University (NTU), Taipei, Taiwan

P. Chang, Y. Chao, K.F. Chen, P.H. Chen, W.-S. Hou, Y.y. Li, R.-S. Lu, E. Paganis, A. Psallidas, A. Steen

Chulalongkorn University, Faculty of Science, Department of Physics, Bangkok, Thailand

B. Asavapibhop, C. Asawatangtrakuldee, N. Srimanobhas, N. Suwonjandee

Çukurova University, Physics Department, Science and Art Faculty, Adana, Turkey

A. Bat, F. Boran, S. Damarseckin ${ }^{51}$, Z.S. Demiroglu, F. Dolek, C. Dozen, G. Gokbulut, EmineGurpinar Guler ${ }^{52}$, Y. Guler, I. Hos ${ }^{53}$, C. Isik, E.E. Kangal ${ }^{54}$, O. Kara, A. Kayis Topaksu, U. Kiminsu, M. Oglakci, G. Onengut, K. Ozdemir ${ }^{55}$, S. Ozturk ${ }^{56}$, A. Polatoz, A.E. Simsek, D. Sunar Cerci $^{57}$, B. Tali ${ }^{57}$, U.G. Tok, S. Turkcapar, I.S. Zorbakir, C. Zorbilmez

Middle East Technical University, Physics Department, Ankara, Turkey

B. Isildak ${ }^{58}$, G. Karapinar ${ }^{59}$, M. Yalvac

Bogazici University, Istanbul, Turkey

I.O. Atakisi, E. Gülmez, M. Kaya ${ }^{60}$, O. Kaya ${ }^{61}$, B. Kaynak, Ö. Özçelik, S. Tekten, E.A. Yetkin ${ }^{62}$

Istanbul Technical University, Istanbul, Turkey

A. Cakir, K. Cankocak, Y. Komurcu, S. Sen ${ }^{63}$

Istanbul University, Istanbul, Turkey

S. Ozkorucuklu

Institute for Scintillation Materials of National Academy of Science of Ukraine, Kharkov, Ukraine

B. Grynyov

National Scientific Center, Kharkov Institute of Physics and Technology, Kharkov, Ukraine

L. Levchuk

University of Bristol, Bristol, United Kingdom

F. Ball, E. Bhal, S. Bologna, J.J. Brooke, D. Burns, E. Clement, D. Cussans, H. Flacher,

J. Goldstein, G.P. Heath, H.F. Heath, L. Kreczko, S. Paramesvaran, B. Penning,

T. Sakuma, S. Seif El Nasr-Storey, D. Smith, V.J. Smith, J. Taylor, A. Titterton

Rutherford Appleton Laboratory, Didcot, United Kingdom

K.W. Bell, A. Belyaev ${ }^{64}$, C. Brew, R.M. Brown, D. Cieri, D.J.A. Cockerill, J.A. Coughlan,

K. Harder, S. Harper, J. Linacre, K. Manolopoulos, D.M. Newbold, E. Olaiya, D. Petyt,

T. Reis, T. Schuh, C.H. Shepherd-Themistocleous, A. Thea, I.R. Tomalin, T. Williams, W.J. Womersley 


\section{Imperial College, London, United Kingdom}

R. Bainbridge, P. Bloch, J. Borg, S. Breeze, O. Buchmuller, A. Bundock, GurpreetSingh CHAHAL ${ }^{65}$, D. Colling, P. Dauncey, G. Davies, M. Della Negra, R. Di Maria, P. Everaerts, G. Hall, G. Iles, T. James, M. Komm, C. Laner, L. Lyons, A.-M. Magnan, S. Malik, A. Martelli, V. Milosevic, J. Nash ${ }^{66}$, V. Palladino, M. Pesaresi, D.M. Raymond, A. Richards, A. Rose, E. Scott, C. Seez, A. Shtipliyski, M. Stoye, T. Strebler, S. Summers, A. Tapper, K. Uchida, T. Virdee ${ }^{17}$, N. Wardle, D. Winterbottom, J. Wright, A.G. Zecchinelli, S.C. Zenz

\section{Brunel University, Uxbridge, United Kingdom}

J.E. Cole, P.R. Hobson, A. Khan, P. Kyberd, C.K. Mackay, A. Morton, I.D. Reid, L. Teodorescu, S. Zahid

\section{Baylor University, Waco, U.S.A.}

K. Call, J. Dittmann, K. Hatakeyama, C. Madrid, B. McMaster, N. Pastika, C. Smith

\section{Catholic University of America, Washington, DC, U.S.A.}

R. Bartek, A. Dominguez, R. Uniyal

\section{The University of Alabama, Tuscaloosa, U.S.A.}

A. Buccilli, S.I. Cooper, C. Henderson, P. Rumerio, C. West

\section{Boston University, Boston, U.S.A.}

D. Arcaro, T. Bose, Z. Demiragli, D. Gastler, S. Girgis, D. Pinna, C. Richardson, J. Rohlf, D. Sperka, I. Suarez, L. Sulak, D. Zou

\section{Brown University, Providence, U.S.A.}

G. Benelli, B. Burkle, X. Coubez, D. Cutts, Y.t. Duh, M. Hadley, J. Hakala, U. Heintz, J.M. Hogan ${ }^{67}$, K.H.M. Kwok, E. Laird, G. Landsberg, J. Lee, Z. Mao, M. Narain, S. Sagir ${ }^{68}$, R. Syarif, E. Usai, D. Yu

University of California, Davis, Davis, U.S.A.

R. Band, C. Brainerd, R. Breedon, M. Calderon De La Barca Sanchez, M. Chertok, J. Conway, R. Conway, P.T. Cox, R. Erbacher, C. Flores, G. Funk, F. Jensen, W. Ko, O. Kukral, R. Lander, M. Mulhearn, D. Pellett, J. Pilot, M. Shi, D. Stolp, D. Taylor, K. Tos, M. Tripathi, Z. Wang, F. Zhang

\section{University of California, Los Angeles, U.S.A.}

M. Bachtis, C. Bravo, R. Cousins, A. Dasgupta, A. Florent, J. Hauser, M. Ignatenko, N. Mccoll, W.A. Nash, S. Regnard, D. Saltzberg, C. Schnaible, B. Stone, V. Valuev

\section{University of California, Riverside, Riverside, U.S.A.}

K. Burt, R. Clare, J.W. Gary, S.M.A. Ghiasi Shirazi, G. Hanson, G. Karapostoli, E. Kennedy, O.R. Long, M. Olmedo Negrete, M.I. Paneva, W. Si, L. Wang, H. Wei, S. Wimpenny, B.R. Yates, Y. Zhang 
University of California, San Diego, La Jolla, U.S.A.

J.G. Branson, P. Chang, S. Cittolin, M. Derdzinski, R. Gerosa, D. Gilbert, B. Hashemi, D. Klein, V. Krutelyov, J. Letts, M. Masciovecchio, S. May, S. Padhi, M. Pieri, V. Sharma, M. Tadel, F. Würthwein, A. Yagil, G. Zevi Della Porta

University of California, Santa Barbara - Department of Physics, Santa Barbara, U.S.A.

N. Amin, R. Bhandari, C. Campagnari, M. Citron, V. Dutta, M. Franco Sevilla, L. Gouskos, J. Incandela, B. Marsh, H. Mei, A. Ovcharova, H. Qu, J. Richman, U. Sarica, D. Stuart, S. Wang, J. Yoo

\section{California Institute of Technology, Pasadena, U.S.A.}

D. Anderson, A. Bornheim, O. Cerri, I. Dutta, J.M. Lawhorn, N. Lu, J. Mao, H.B. Newman, T.Q. Nguyen, J. Pata, M. Spiropulu, J.R. Vlimant, S. Xie, Z. Zhang, R.Y. Zhu

Carnegie Mellon University, Pittsburgh, U.S.A.

M.B. Andrews, T. Ferguson, T. Mudholkar, M. Paulini, M. Sun, I. Vorobiev, M. Weinberg

University of Colorado Boulder, Boulder, U.S.A.

J.P. Cumalat, W.T. Ford, A. Johnson, E. MacDonald, T. Mulholland, R. Patel, A. Perloff, K. Stenson, K.A. Ulmer, S.R. Wagner

Cornell University, Ithaca, U.S.A.

J. Alexander, J. Chaves, Y. Cheng, J. Chu, A. Datta, A. Frankenthal, K. Mcdermott, N. Mirman, J.R. Patterson, D. Quach, A. Rinkevicius ${ }^{69}$, A. Ryd, S.M. Tan, Z. Tao, J. Thom, P. Wittich, M. Zientek

Fermi National Accelerator Laboratory, Batavia, U.S.A.

S. Abdullin, M. Albrow, M. Alyari, G. Apollinari, A. Apresyan, A. Apyan, S. Banerjee, L.A.T. Bauerdick, A. Beretvas, J. Berryhill, P.C. Bhat, K. Burkett, J.N. Butler, A. Canepa, G.B. Cerati, H.W.K. Cheung, F. Chlebana, M. Cremonesi, J. Duarte, V.D. Elvira, J. Freeman, Z. Gecse, E. Gottschalk, L. Gray, D. Green, S. Grünendahl, O. Gutsche, AllisonReinsvold Hall, J. Hanlon, R.M. Harris, S. Hasegawa, R. Heller, J. Hirschauer, B. Jayatilaka, S. Jindariani, M. Johnson, U. Joshi, B. Klima, M.J. Kortelainen, B. Kreis, S. Lammel, J. Lewis, D. Lincoln, R. Lipton, M. Liu, T. Liu, J. Lykken, K. Maeshima, J.M. Marraffino, D. Mason, P. McBride, P. Merkel, S. Mrenna, S. Nahn, V. O’Dell, V. Papadimitriou, K. Pedro, C. Pena, G. Rakness, F. Ravera, L. Ristori, B. Schneider, E. Sexton-Kennedy, N. Smith, A. Soha, W.J. Spalding, L. Spiegel, S. Stoynev, J. Strait, N. Strobbe, L. Taylor, S. Tkaczyk, N.V. Tran, L. Uplegger, E.W. Vaandering, C. Vernieri, M. Verzocchi, R. Vidal, M. Wang, H.A. Weber

\section{University of Florida, Gainesville, U.S.A.}

D. Acosta, P. Avery, P. Bortignon, D. Bourilkov, A. Brinkerhoff, L. Cadamuro, A. Carnes,

V. Cherepanov, D. Curry, F. Errico, R.D. Field, S.V. Gleyzer, B.M. Joshi, M. Kim, J. Konigsberg, A. Korytov, K.H. Lo, P. Ma, K. Matchev, N. Menendez, G. Mitselmakher, D. Rosenzweig, K. Shi, J. Wang, S. Wang, X. Zuo 
Florida International University, Miami, U.S.A.

Y.R. Joshi

Florida State University, Tallahassee, U.S.A.

T. Adams, A. Askew, S. Hagopian, V. Hagopian, K.F. Johnson, R. Khurana, T. Kolberg, G. Martinez, T. Perry, H. Prosper, C. Schiber, R. Yohay, J. Zhang

Florida Institute of Technology, Melbourne, U.S.A.

M.M. Baarmand, V. Bhopatkar, M. Hohlmann, D. Noonan, M. Rahmani, M. Saunders, F. Yumiceva

University of Illinois at Chicago (UIC), Chicago, U.S.A.

M.R. Adams, L. Apanasevich, D. Berry, R.R. Betts, R. Cavanaugh, X. Chen, S. Dittmer, O. Evdokimov, C.E. Gerber, D.A. Hangal, D.J. Hofman, K. Jung, C. Mills, T. Roy, M.B. Tonjes, N. Varelas, H. Wang, X. Wang, Z. Wu

The University of Iowa, Iowa City, U.S.A.

M. Alhusseini, B. Bilki ${ }^{52}$, W. Clarida, K. Dilsiz ${ }^{70}$, S. Durgut, R.P. Gandrajula, M. Haytmyradov, V. Khristenko, O.K. Köseyan, J.-P. Merlo, A. Mestvirishvili ${ }^{71}$, A. Moeller, J. Nachtman, H. Ogul ${ }^{72}$, Y. Onel, F. Ozok ${ }^{73}$, A. Penzo, C. Snyder, E. Tiras, J. Wetzel

Johns Hopkins University, Baltimore, U.S.A.

B. Blumenfeld, A. Cocoros, N. Eminizer, D. Fehling, L. Feng, A.V. Gritsan, W.T. Hung, P. Maksimovic, J. Roskes, M. Swartz, M. Xiao

The University of Kansas, Lawrence, U.S.A.

C. Baldenegro Barrera, P. Baringer, A. Bean, S. Boren, J. Bowen, A. Bylinkin, T. Isidori, S. Khalil, J. King, G. Krintiras, A. Kropivnitskaya, C. Lindsey, D. Majumder, W. Mcbrayer, N. Minafra, M. Murray, C. Rogan, C. Royon, S. Sanders, E. Schmitz, J.D. Tapia Takaki, Q. Wang, J. Williams, G. Wilson

Kansas State University, Manhattan, U.S.A.

S. Duric, A. Ivanov, K. Kaadze, D. Kim, Y. Maravin, D.R. Mendis, T. Mitchell, A. Modak, A. Mohammadi

Lawrence Livermore National Laboratory, Livermore, U.S.A.

F. Rebassoo, D. Wright

University of Maryland, College Park, U.S.A.

A. Baden, O. Baron, A. Belloni, S.C. Eno, Y. Feng, N.J. Hadley, S. Jabeen, G.Y. Jeng, R.G. Kellogg, J. Kunkle, A.C. Mignerey, S. Nabili, F. Ricci-Tam, M. Seidel, Y.H. Shin, A. Skuja, S.C. Tonwar, K. Wong

Massachusetts Institute of Technology, Cambridge, U.S.A.

D. Abercrombie, B. Allen, A. Baty, R. Bi, S. Brandt, W. Busza, I.A. Cali, M. D'Alfonso, G. Gomez Ceballos, M. Goncharov, P. Harris, D. Hsu, M. Hu, M. Klute, D. Kovalskyi, Y.-J. Lee, P.D. Luckey, B. Maier, A.C. Marini, C. Mcginn, C. Mironov, S. Narayanan, X. Niu, C. Paus, D. Rankin, C. Roland, G. Roland, Z. Shi, G.S.F. Stephans, K. Sumorok, K. Tatar, D. Velicanu, J. Wang, T.W. Wang, B. Wyslouch 
University of Minnesota, Minneapolis, U.S.A.

A.C. Benvenuti ${ }^{\dagger}$, R.M. Chatterjee, A. Evans, S. Guts, P. Hansen, J. Hiltbrand, Sh. Jain, S. Kalafut, Y. Kubota, Z. Lesko, J. Mans, R. Rusack, M.A. Wadud

University of Mississippi, Oxford, U.S.A.

J.G. Acosta, S. Oliveros

University of Nebraska-Lincoln, Lincoln, U.S.A.

K. Bloom, D.R. Claes, C. Fangmeier, L. Finco, F. Golf, R. Gonzalez Suarez, R. Kamalieddin, I. Kravchenko, J.E. Siado, G.R. Snow, B. Stieger

State University of New York at Buffalo, Buffalo, U.S.A.

G. Agarwal, C. Harrington, I. Iashvili, A. Kharchilava, C. Mclean, D. Nguyen, A. Parker, J. Pekkanen, S. Rappoccio, B. Roozbahani

Northeastern University, Boston, U.S.A.

G. Alverson, E. Barberis, C. Freer, Y. Haddad, A. Hortiangtham, G. Madigan, D.M. Morse,

T. Orimoto, L. Skinnari, A. Tishelman-Charny, T. Wamorkar, B. Wang, A. Wisecarver,

D. Wood

Northwestern University, Evanston, U.S.A.

S. Bhattacharya, J. Bueghly, T. Gunter, K.A. Hahn, N. Odell, M.H. Schmitt, K. Sung, M. Trovato, M. Velasco

University of Notre Dame, Notre Dame, U.S.A.

R. Bucci, N. Dev, R. Goldouzian, M. Hildreth, K. Hurtado Anampa, C. Jessop, D.J. Karmgard, K. Lannon, W. Li, N. Loukas, N. Marinelli, I. Mcalister, F. Meng, C. Mueller, Y. Musienko ${ }^{36}$, M. Planer, R. Ruchti, P. Siddireddy, G. Smith, S. Taroni, M. Wayne, A. Wightman, M. Wolf, A. Woodard

The Ohio State University, Columbus, U.S.A.

J. Alimena, B. Bylsma, L.S. Durkin, S. Flowers, B. Francis, C. Hill, W. Ji, A. Lefeld, T.Y. Ling, B.L. Winer

Princeton University, Princeton, U.S.A.

S. Cooperstein, G. Dezoort, P. Elmer, J. Hardenbrook, N. Haubrich, S. Higginbotham, A. Kalogeropoulos, S. Kwan, D. Lange, M.T. Lucchini, J. Luo, D. Marlow, K. Mei, I. Ojalvo, J. Olsen, C. Palmer, P. Piroué, J. Salfeld-Nebgen, D. Stickland, C. Tully, Z. Wang

University of Puerto Rico, Mayaguez, U.S.A.

S. Malik, S. Norberg

Purdue University, West Lafayette, U.S.A.

A. Barker, V.E. Barnes, S. Das, L. Gutay, M. Jones, A.W. Jung, A. Khatiwada, B. Mahakud, D.H. Miller, G. Negro, N. Neumeister, C.C. Peng, S. Piperov, H. Qiu, J.F. Schulte, J. Sun, F. Wang, R. Xiao, W. Xie

Purdue University Northwest, Hammond, U.S.A.

T. Cheng, J. Dolen, N. Parashar 
Rice University, Houston, U.S.A.

K.M. Ecklund, S. Freed, F.J.M. Geurts, M. Kilpatrick, Arun Kumar, W. Li, B.P. Padley, R. Redjimi, J. Roberts, J. Rorie, W. Shi, A.G. Stahl Leiton, Z. Tu, A. Zhang

University of Rochester, Rochester, U.S.A.

A. Bodek, P. de Barbaro, R. Demina, J.L. Dulemba, C. Fallon, T. Ferbel, M. Galanti, A. Garcia-Bellido, J. Han, O. Hindrichs, A. Khukhunaishvili, E. Ranken, P. Tan, R. Taus

Rutgers, The State University of New Jersey, Piscataway, U.S.A.

B. Chiarito, J.P. Chou, A. Gandrakota, Y. Gershtein, E. Halkiadakis, A. Hart, M. Heindl, E. Hughes, S. Kaplan, S. Kyriacou, I. Laflotte, A. Lath, R. Montalvo, K. Nash, M. Osherson, H. Saka, S. Salur, S. Schnetzer, D. Sheffield, S. Somalwar, R. Stone, S. Thomas, P. Thomassen

University of Tennessee, Knoxville, U.S.A.

H. Acharya, A.G. Delannoy, J. Heideman, G. Riley, S. Spanier

Texas A\&M University, College Station, U.S.A.

O. Bouhali ${ }^{74}$, A. Celik, M. Dalchenko, M. De Mattia, A. Delgado, S. Dildick, R. Eusebi, J. Gilmore, T. Huang, T. Kamon ${ }^{75}$, S. Luo, D. Marley, R. Mueller, D. Overton, L. Perniè, D. Rathjens, A. Safonov

Texas Tech University, Lubbock, U.S.A.

N. Akchurin, J. Damgov, F. De Guio, S. Kunori, K. Lamichhane, S.W. Lee, T. Mengke, S. Muthumuni, T. Peltola, S. Undleeb, I. Volobouev, Z. Wang, A. Whitbeck

Vanderbilt University, Nashville, U.S.A.

S. Greene, A. Gurrola, R. Janjam, W. Johns, C. Maguire, A. Melo, H. Ni, K. Padeken, F. Romeo, P. Sheldon, S. Tuo, J. Velkovska, M. Verweij

University of Virginia, Charlottesville, U.S.A.

M.W. Arenton, P. Barria, B. Cox, G. Cummings, R. Hirosky, M. Joyce, A. Ledovskoy, C. Neu, B. Tannenwald, Y. Wang, E. Wolfe, F. Xia

Wayne State University, Detroit, U.S.A.

R. Harr, P.E. Karchin, N. Poudyal, J. Sturdy, P. Thapa, S. Zaleski

University of Wisconsin - Madison, Madison, WI, U.S.A.

J. Buchanan, C. Caillol, D. Carlsmith, S. Dasu, I. De Bruyn, L. Dodd, F. Fiori, C. Galloni, B. Gomber ${ }^{76}$, M. Herndon, A. Hervé, U. Hussain, P. Klabbers, A. Lanaro, A. Loeliger, K. Long, R. Loveless, J. Madhusudanan Sreekala, T. Ruggles, A. Savin, V. Sharma, W.H. Smith, D. Teague, S. Trembath-reichert, N. Woods

$\dagger$ : Deceased

1: Also at Vienna University of Technology, Vienna, Austria

2: Also at IRFU, CEA, Université Paris-Saclay, Gif-sur-Yvette, France

3: Also at Universidade Estadual de Campinas, Campinas, Brazil

4: Also at Federal University of Rio Grande do Sul, Porto Alegre, Brazil 
5: Also at UFMS, Nova Andradina, Brazil

6: Also at Universidade Federal de Pelotas, Pelotas, Brazil

7: Also at Université Libre de Bruxelles, Bruxelles, Belgium

8: Also at University of Chinese Academy of Sciences, Beijing, China

9: Also at Institute for Theoretical and Experimental Physics named by A.I. Alikhanov of NRC 'Kurchatov Institute', Moscow, Russia

10: Also at Joint Institute for Nuclear Research, Dubna, Russia

11: Also at Cairo University, Cairo, Egypt

12: Also at Helwan University, Cairo, Egypt

13: Now at Zewail City of Science and Technology, Zewail, Egypt

14: Also at Purdue University, West Lafayette, U.S.A.

15: Also at Université de Haute Alsace, Mulhouse, France

16: Also at Erzincan Binali Yildirim University, Erzincan, Turkey

17: Also at CERN, European Organization for Nuclear Research, Geneva, Switzerland

18: Also at RWTH Aachen University, III. Physikalisches Institut A, Aachen, Germany

19: Also at University of Hamburg, Hamburg, Germany

20: Also at Brandenburg University of Technology, Cottbus, Germany

21: Also at Institute of Physics, University of Debrecen, Debrecen, Hungary, Debrecen, Hungary

22: Also at Institute of Nuclear Research ATOMKI, Debrecen, Hungary

23: Also at MTA-ELTE Lendület CMS Particle and Nuclear Physics Group, Eötvös Loránd University, Budapest, Hungary, Budapest, Hungary

24: Also at IIT Bhubaneswar, Bhubaneswar, India, Bhubaneswar, India

25: Also at Institute of Physics, Bhubaneswar, India

26: Also at Shoolini University, Solan, India

27: Also at University of Visva-Bharati, Santiniketan, India

28: Also at Isfahan University of Technology, Isfahan, Iran

29: Also at Italian National Agency for New Technologies, Energy and Sustainable Economic Development, Bologna, Italy

30: Also at Centro Siciliano di Fisica Nucleare e di Struttura Della Materia, Catania, Italy

31: Also at Scuola Normale e Sezione dell'INFN, Pisa, Italy

32: Also at Riga Technical University, Riga, Latvia, Riga, Latvia

33: Also at Malaysian Nuclear Agency, MOSTI, Kajang, Malaysia

34: Also at Consejo Nacional de Ciencia y Tecnología, Mexico City, Mexico

35: Also at Warsaw University of Technology, Institute of Electronic Systems, Warsaw, Poland

36: Also at Institute for Nuclear Research, Moscow, Russia

37: Now at National Research Nuclear University 'Moscow Engineering Physics Institute' (MEPhI), Moscow, Russia

38: Also at Institute of Nuclear Physics of the Uzbekistan Academy of Sciences, Tashkent, Uzbekistan

39: Also at St. Petersburg State Polytechnical University, St. Petersburg, Russia

40: Also at University of Florida, Gainesville, U.S.A.

41: Also at Imperial College, London, United Kingdom

42: Also at P.N. Lebedev Physical Institute, Moscow, Russia

43: Also at California Institute of Technology, Pasadena, U.S.A.

44: Also at Budker Institute of Nuclear Physics, Novosibirsk, Russia

45: Also at Faculty of Physics, University of Belgrade, Belgrade, Serbia

46: Also at Università degli Studi di Siena, Siena, Italy

47: Also at INFN Sezione di Pavia ${ }^{a}$, Università di Pavia ${ }^{b}$, Pavia, Italy, Pavia, Italy 
48: Also at National and Kapodistrian University of Athens, Athens, Greece

49: Also at Universität Zürich, Zurich, Switzerland

50: Also at Stefan Meyer Institute for Subatomic Physics, Vienna, Austria, Vienna, Austria

51: Also at Sirnak University, Sirnak, Turkey

52: Also at Beykent University, Istanbul, Turkey, Istanbul, Turkey

53: Also at Istanbul Aydin University, Istanbul, Turkey

54: Also at Mersin University, Mersin, Turkey

55: Also at Piri Reis University, Istanbul, Turkey

56: Also at Gaziosmanpasa University, Tokat, Turkey

57: Also at Adiyaman University, Adiyaman, Turkey

58: Also at Ozyegin University, Istanbul, Turkey

59: Also at Izmir Institute of Technology, Izmir, Turkey

60: Also at Marmara University, Istanbul, Turkey

61: Also at Kafkas University, Kars, Turkey

62: Also at Istanbul Bilgi University, Istanbul, Turkey

63: Also at Hacettepe University, Ankara, Turkey

64: Also at School of Physics and Astronomy, University of Southampton, Southampton, United Kingdom

65: Also at IPPP Durham University, Durham, United Kingdom

66: Also at Monash University, Faculty of Science, Clayton, Australia

67: Also at Bethel University, St. Paul, Minneapolis, U.S.A., St. Paul, U.S.A.

68: Also at Karamanoğlu Mehmetbey University, Karaman, Turkey

69: Also at Vilnius University, Vilnius, Lithuania

70: Also at Bingol University, Bingol, Turkey

71: Also at Georgian Technical University, Tbilisi, Georgia

72: Also at Sinop University, Sinop, Turkey

73: Also at Mimar Sinan University, Istanbul, Istanbul, Turkey

74: Also at Texas A\&M University at Qatar, Doha, Qatar

75: Also at Kyungpook National University, Daegu, Korea, Daegu, Korea

76: Also at University of Hyderabad, Hyderabad, India 